Article

\title{
Development of a Sensitive and Reliable UHPLC-MS/MS Method for the Determination of Multiple Urinary Biomarkers of Mycotoxin Exposure
}

\author{
Zhezhe Liu ${ }^{1,2}{ }^{\text {, Xiaoxue Zhao }}{ }^{2}$, Libiao Wu ${ }^{1}$, Shuang Zhou ${ }^{2, *}$, Zhiyong Gong ${ }^{1}$, Yunfeng Zhao ${ }^{2}$ \\ and Yongning $\mathrm{Wu}^{2, * \mathbb{D}}$ \\ 1 School of Food Science and Engineering, Wuhan Polytechnic University, Wuhan 430023, China; \\ liuzhzh968@hotmail.com (Z.L.); wulibiaowhpu@hotmail.com (L.W.); gongzycn@whpu.edu.cn (Z.G.) \\ 2 NHC Key Laboratory of Food Safety Risk Assessment, Food Safety Research Unit (2019RU014) of Chinese \\ Academy of Medical Science, China National Center for Food Safety Risk Assessment, Beijing 100021, China; \\ zhaoxx@cfsa.net.cn (X.Z.); zhaoyf@cfsa.net.cn (Y.Z.) \\ * Correspondence: zhoush@cfsa.net.cn (S.Z.); wuyongning@cfsa.net.cn (Y.W.); Tel.: +86-10-6777-9768 (S.Z.); \\ +86-10-5216-5589 (Y.W.)
}

Received: 13 February 2020; Accepted: 15 March 2020; Published: 18 March 2020

\begin{abstract}
A variety of mycotoxins from different sources frequently contaminate farm products, presenting a potential toxicological concern for animals and human. Mycotoxin exposure has been the focus of attention for governments around the world. To date, biomarkers are used to monitor mycotoxin exposure and promote new understanding of their role in chronic diseases. The goal of this research was to develop and validate a sensitive UHPLC-MS/MS method using isotopically-labeled internal standards suitable for accurate determination of 18 mycotoxin biomarkers, including fumonisins, ochratoxins, Alternaria and emerging Fusarium mycotoxins (fumonisin $\mathrm{B}_{1}$, $\mathrm{B}_{2}$, and $\mathrm{B}_{3}$, hydrolyzed fumonisin $\mathrm{B}_{1}$ and $\mathrm{B}_{2}$, ochratoxin $\mathrm{A}, \mathrm{B}$, and alpha, alternariol, alternariol monomethyl ether, altenuene, tentoxin, tenuazonic acid, beauvericin, enniatin $A, A_{1}, B$, and $B_{1}$ ) in human urine. After enzymatic digestion with $\beta$-glucuronidase, human urine samples were cleaned up using HLB solid phase extraction cartridges prior to instrument analysis. The multi-mycotoxin and analyte-specific method was validated in-house, providing satisfactory results. The method provided good linearity in the tested concentration range (from LOQ up to $25-500 \mathrm{ng} / \mathrm{mL}$ for different analytes), with $R^{2}$ from 0.997 to 0.999 . The limits of quantitation varied from 0.0002 to $0.5 \mathrm{ng} / \mathrm{mL}$ for all analytes in urine. The recoveries for spiked samples were between $74.0 \%$ and $133 \%$, with intra-day precision of $0.5 \%-8.7 \%$ and inter-day precision of $2.4 \%-13.4 \%$. This method was applied to 60 urine samples collected from healthy volunteers in Beijing, and 10 biomarkers were found. At least one biomarker was found in all but one of the samples. The high sensitivity and accuracy of this method make it practical for human biomonitoring and mycotoxin exposure assessment.
\end{abstract}

Keywords: human biomonitoring; emerging mycotoxins; metabolites; biomarkers; urine; UHPLC-MS/MS

Key Contribution: A reliable and sensitive UHPLC-MS/MS method for quantification of 18 mycotoxins and their metabolites $\left(\mathrm{FB}_{1} ; \mathrm{FB}_{2} ; \mathrm{FB}_{3} ; \mathrm{HFB}_{1} ; \mathrm{HFB}_{2} ; \mathrm{OTA} ; \mathrm{OTB} ; \mathrm{OT}\right.$-alpha; TeA; ALT; AME; $\mathrm{AOH}$; TEN; $\mathrm{BEA}$; ENNA; $\mathrm{ENNA}_{1}$; $\mathrm{ENNB}$; and $\mathrm{ENNB}_{1}$ ) in human urine was developed and validated. The method is applicable for human biomonitoring and multi-mycotoxin exposure study. 


\section{Introduction}

Mycotoxins are toxic secondary metabolites produced under favorable conditions by different species of fungi that can grow in a wide variety of cereals and foods from production to storage $[1,2]$. Their occurrence and concentrations vary considerably among foods due to factors such as crop susceptibility, climate change, storage and transportation conditions, as well as sanitary standards $[3,4]$. Mycotoxins are hepatotoxic, nephrotoxic, teratogenic, carcinogenic, cytotoxic, immunosuppressive, inflammatory, neurotoxic, and estrogenic, posing diverse health hazards to humans and animals [5-8]. In particular, fumonisins (FBs) and ochratoxin a (OTA) have been classified as possible human carcinogens (Group 2B). These major mycotoxins have been strictly regulated by the Joint FAO/WHO Expert Committee on Food Additives (JECFA), and many countries have set maximum levels (MLs) in food and feed $[9,10]$.

With climatic change, some emerging mycotoxins, Alternaria toxins, and Fusarium mycotoxins (enniatins and beauvericin) have been found in foods. Alternaria toxins, including alternariol (AOH), alternariol monomethyl ether (AME), altenuene (ALT), tentoxin (TEN), and tenuazonic acid (TeA), are produced by Alternaria species and have strong evidence of acute and chronic toxicity [11]. The most well-known enniatins (ENNs) reported as natural contaminants are ENNA, ENNA1, ENNB, and ENNB1, which have shown cytotoxic and apoptotic activities [12]. Studies published to date have rarely paid close attention to Alternaria and emerging Fusarium mycotoxins, despite several scientific reports published by the European Food Safety Authority (EFSA) [11,12]. Regulations or MLs for these emerging toxins in food and feed have not been established due to the absence of comprehensive occurrence data and toxicological characterization. Therefore, it is imperative to collect their occurrence and exposure data worldwide.

Mycotoxin exposure assessment is traditionally based on calculations combining mycotoxin contamination in food data with population food consumption data [13]. Taking into account the heterogeneous distribution of mycotoxins in food, the modified forms that cannot be determined, and the sources other than dietary intake (i.e., inhalation and occupational exposure), human biomonitoring (HBM) using biomarkers of exposure in biological fluids has been accepted as a suitable alternative to assess the aggregated exposure to mycotoxins from different origins for a more accurate and comprehensive assessment at the national, regional, or even individual levels [14-16]. Exposure biomarkers of mycotoxins include the parent compounds themselves, the metabolites formed in vivo or products that interact with macromolecules, such as DNA or proteins [17]. Urine, faeces, blood, breast milk, or hair may be selected as biological samples for biomarker analysis. Urine is the preferred sample matrix, as it is easy to collect, readily available, and easy to handle. Urinary biomarkers usually provide information about recent intake, especially for mycotoxins possessing high excretion rates through the kidneys [18].

Non-metabolized fumonisins $\mathrm{B}_{1}\left(\mathrm{FB}_{1}\right)$ and ochratoxin a (OTA) are important urinary biomarkers [19]. Moreover, ochratoxin alpha (OT-alpha) is also a well-known metabolite of OTA generated by segmentation of the peptide bond of OTA in vitro [20]. Muñoz et al. suggested that OT-alpha could be a sensitive biomarker [21]. Both OTA and OT-alpha are excreted mainly by urine in humans [20]. Fumonisin $\mathrm{B}_{1}\left(\mathrm{FB}_{1}\right)$, fumonisin $\mathrm{B}_{2}\left(\mathrm{FB}_{2}\right)$, and fumonisin $\mathrm{B}_{3}\left(\mathrm{FB}_{3}\right)$ are naturally present in corn or corn-based products [22]. Fumonisins are highly stable in vivo and are mainly excreted via the fecal route, with less than $3 \%$ recovered in urine [23-25]. a portion of fumonisins can also be degraded to hydrolyzed fumonisins in human microsomes [26]. Hydrolyzed fumonisin $\mathrm{B}_{1}\left(\mathrm{HFB}_{1}\right)$ has been found in the gut of vervet monkeys and can be used as an additional biomarker $[27,28]$.

The urinary excretion of TeA was confirmed to be close to $100 \%$. Some studies concluded that efficient urinary excretion of $\mathrm{AOH}$ and $\mathrm{AME}$ were $9 \%$ and over $2.6 \%$ after $24 \mathrm{~h}$, respectively. $\mathrm{AOH}$ has been used as a biomarker of exposure in human urine samples [3]. Some cell and animal experiments identified the metabolites of $\mathrm{AOH}$ and $\mathrm{AME}$ to be $\mathrm{AOH}-3 \mathrm{GlcA}, \mathrm{AOH}-7 \mathrm{GlcA}, \mathrm{AOH}-9 \mathrm{GlcA}, \mathrm{AME}-3 \mathrm{GlcA}$, and AME-7GlcA [11]. ENNs and beauvericin (BEA) are bioactive compounds. For ENNs, since the 
information about their metabolism is still scarce, determination of parent compounds is the current evaluation method [7].

According to the description above, mycotoxin biomarkers are excreted in urine in the form of free and conjugated forms. Generally, conjugated forms cannot be quantified directly because of the lack of standard materials [29]. Glucuronidation is one of the main phase II metabolic pathways in the human body. Mycotoxin-glucuronides can be formed in liver and excreted in urine [30]. Hence, in this study, urine samples were digested with $\beta$-glucuronidase to break down the conjugated forms and obtain more accurate exposure results.

Among the published methods for mycotoxin determination in biological samples, including HPLC [20,31,32], GC-MS/MS [33], LC-MS [34,35], LC-MS/MS [4,18,36,37], and LC-HRMS [38], LC-MS/MS provides remarkable selectivity, accuracy and sensitivity. Most of the methods were applied to detect common regulated mycotoxins [4,5,8,19,32,37,39-42], employing various sample preparation strategies such as the "dilute and shoot" approach [4,5,37,41], QuEChERS [15], liquid-liquid extraction (LLE) [20], immunoaffinity (IAC) columns [41,42], solid phase extraction (SPE) [18], and various combinations of these techniques [13]. However, only a few methods targeted for Alternaria and emerging Fusarium mycotoxins, including ENNB [38,43], AOH [3], AOH and AME [15], TeA [44], ENNs, and BEA [7], for human biomonitoring.

To expand the research scope, acquire more extensive occurrence data about multiple mycotoxins and characterize their potential risks, we developed a method for simultaneous quantitation of 18 mycotoxin biomarkers, including common toxins and emerging toxins in urine. The method provided satisfactory recovery and precision for human biomonitoring. Isotope internal standards were used to ensure accuracy and effectively compensate for changes in matrix effects, extraction, and even other unperceived potential interference problems. Furthermore, this method covered a major category of mycotoxin metabolites, the glucuronide conjugates, by using $\beta$-glucuronidase to release them into free forms.

\section{Results and Discussion}

\subsection{Sample Clean-Up}

When simultaneously determining multiple target analytes, sample pretreatment steps are critical to overcome matrix interference, and this process can improve method recovery and sensitivity in UHPLC-MS/MS detection at the same time, especially for complex samples such as urine and serum. Urine contains many impurities that vary in composition and concentration. Several cartridges, such as Mycosep 226, Multisep ${ }^{\circledR} 211$ Fum, and Oasis HLB, have been successfully applied to mycotoxin analyses in food, feed, serum, and urine. Mycosep and Multisep cartridges are multi-functional columns containing a combination of adsorbents that was specifically designed for mycotoxin analysis. These cartridges can retain interfering substances from complex samples and allow analytes of interest to pass through.

Hence, these cartridges were evaluated in this work. a working solution of standard mixture was used to compare the recoveries of the target analytes. Figure 1 shows the percent recovery obtained from different cartridges for 8 analytes (ochratoxins and fumonisins). Other 10 analytes (Alternaria toxins, ENNs and BEA) were optimized in our laboratory, and the results were published previously [45]. We compared the performance of Mycosep 226, Oasis C18 cartridges, and Oasis HLB and their ability to enrich the 10 compounds. Better recoveries were obtained when using the Oasis HLB column.

Under normal dietary conditions, the $\mathrm{pH}$ of human urine is 4.6-8.0. According to the instructions of the HLB column, samples were acidified with formic acid before loading. Then, the $\mathrm{pH}$ value of loading sample was optimized to obtain maximum and stable retention of 18 analytes on the HLB cartridge. As shown in Figure 2, $\mathrm{pH} 3$ gave a satisfactory result. At $\mathrm{pH}$ 6, TeA is in an ionic state and cannot be well retained by the HLB cartridge. 


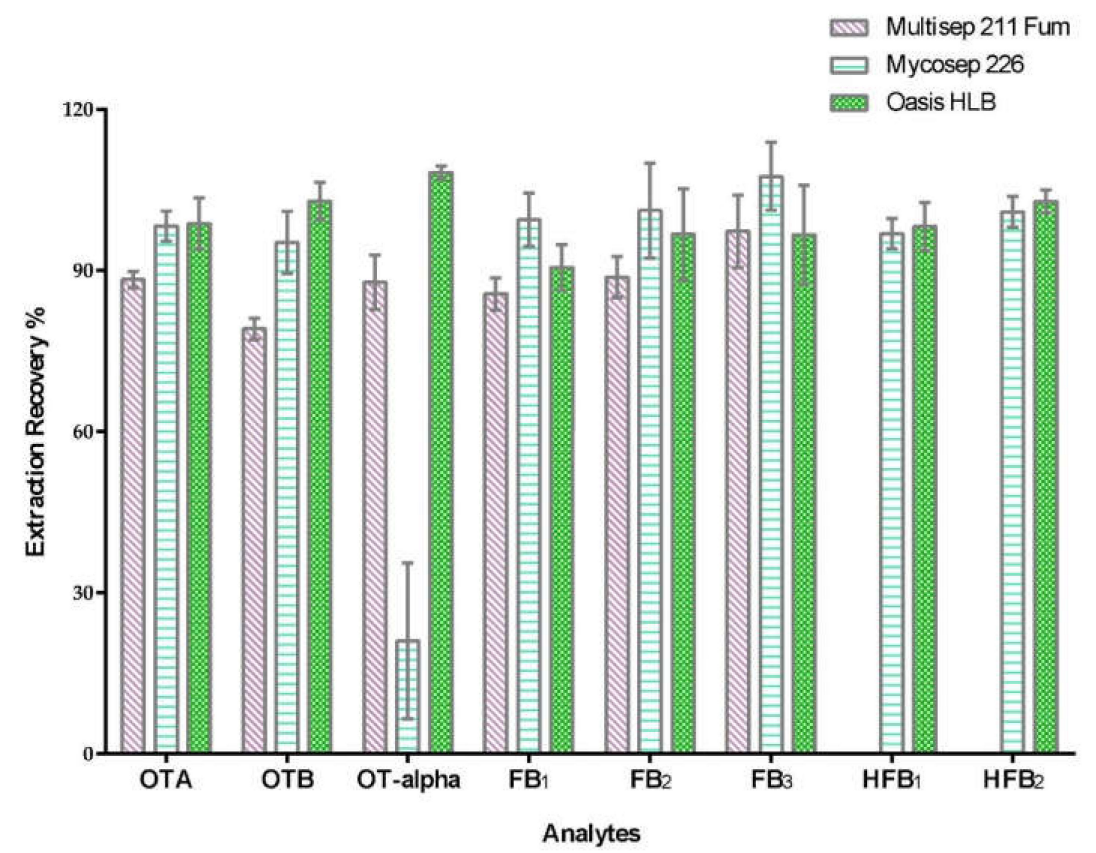

Figure 1. Extraction recovery using Multisep 211 Fum, Mycosep 226 and Oasis HLB cartridge for 8 mycotoxins (OTA, OTB, OT-alpha, $\mathrm{FB}_{1}, \mathrm{FB}_{2}, \mathrm{FB}_{3}, \mathrm{HFB}_{1}$, and $\mathrm{HFB}_{2}$ ) spiked at $10 \mathrm{ng} / \mathrm{mL}$.

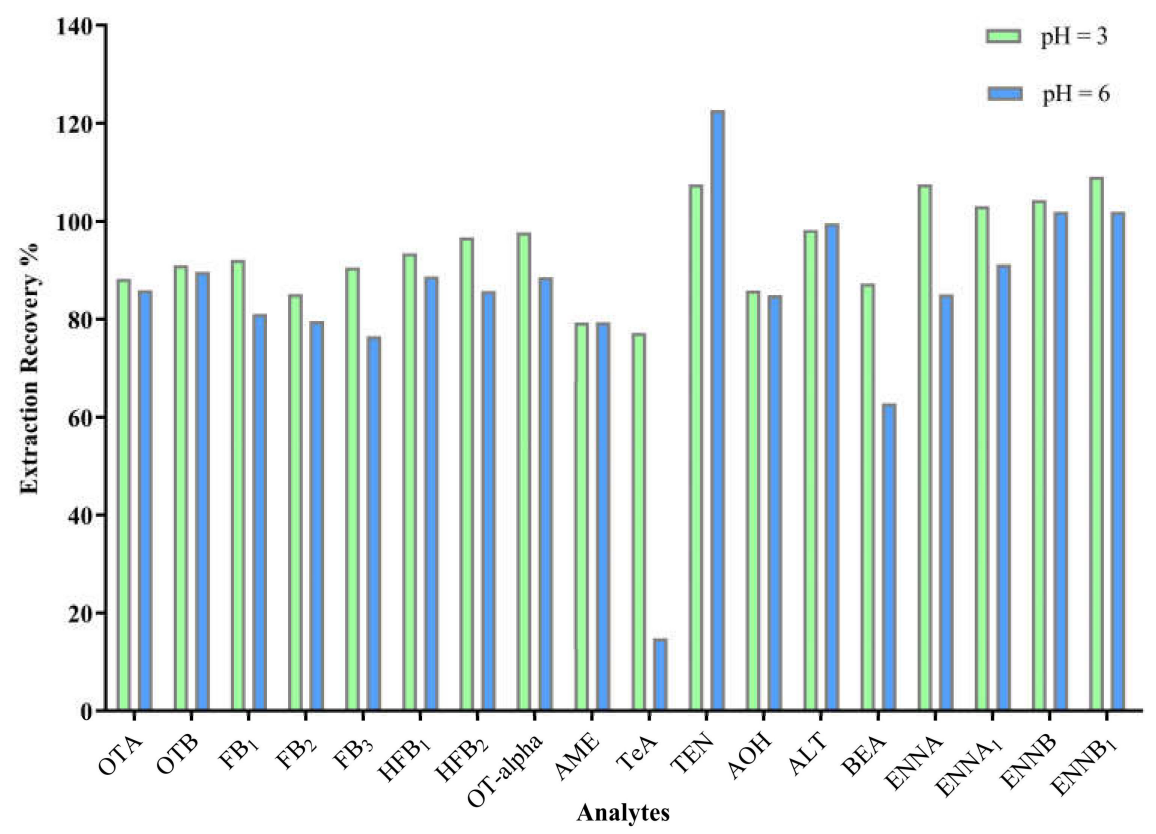

Figure 2. Extraction recovery using Oasis HLB cartridge at different $\mathrm{pH}$ values for 18 target mycotoxins spiked at $10 \mathrm{ng} / \mathrm{mL}$.

Urine contains multiple endogenous water-soluble components, including mineral salts, hormones, vitamins, amino acids, urea, creatinine, and other metabolites, which may cause complex interferences and column clogging. As shown in Figure 3, different eluents $(20 \% \mathrm{MeOH}, 50 \% \mathrm{MeOH}, 80 \% \mathrm{MeOH}$, $100 \% \mathrm{MeOH}$ and equal proportion of $\mathrm{MeOH}$ and $\mathrm{ACN}, \mathrm{v} / \mathrm{v}$ ) were tested. The solution containing $20 \% \mathrm{MeOH}$ was selected as the wash solution to reduce impurities and increase analyte recovery. Five milliliters of $\mathrm{MeOH}$ and then $5 \mathrm{~mL}$ of $\mathrm{ACN}$ were used as the eluent, to obtain the highest recovery 
for most target compounds. The eluate was dried under nitrogen gas and reconstituted in $0.2 \%$ FAc/ACN (50/50, v/v).

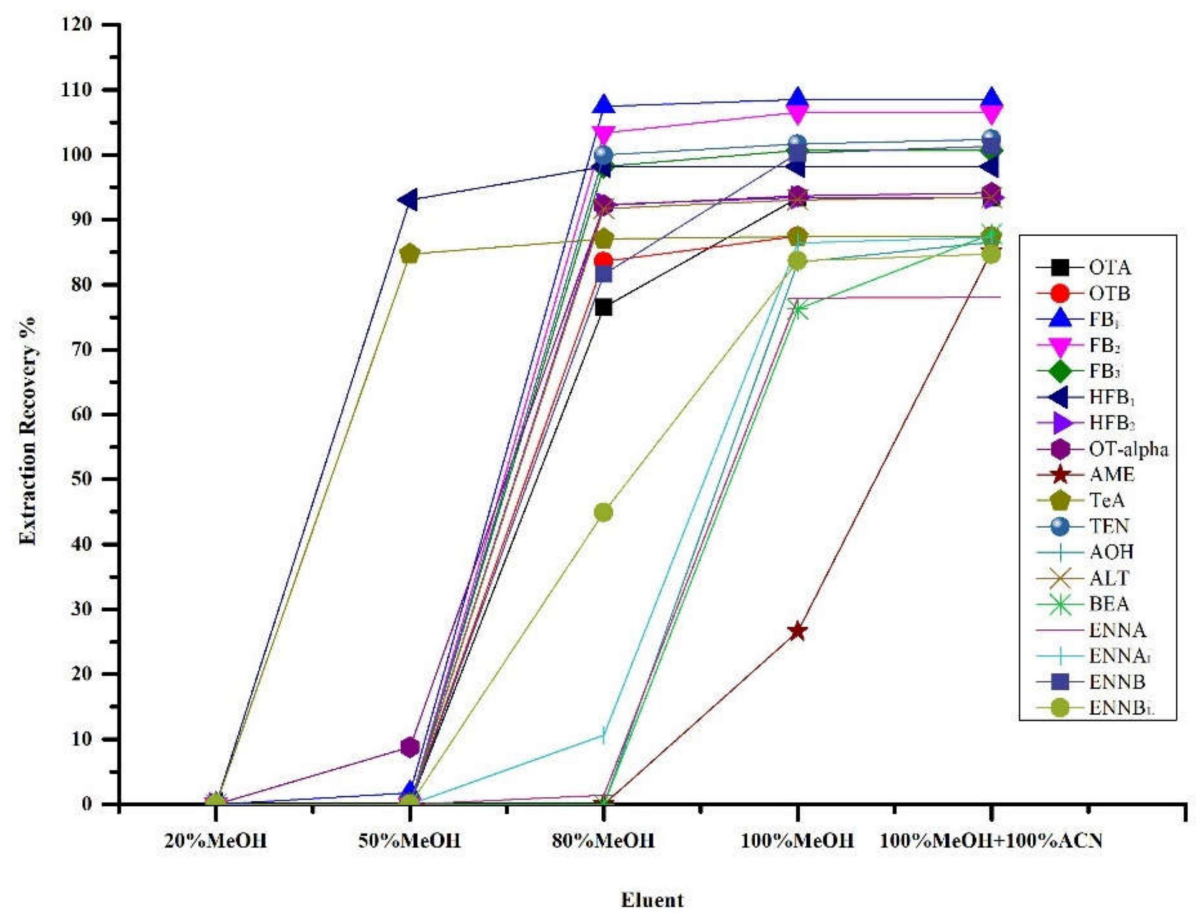

Figure 3. Recovery using different eluent for 18 mycotoxins spiked at $10 \mathrm{ng} / \mathrm{mL}$.

\subsection{Enzyme Hydrolysis}

After ingestion, a portion of mycotoxin can be metabolized to glucuronides and excreted into urine. To obtain the total (free + glucuronides) level of urinary biomarkers, an enzyme hydrolysis using $\beta$-glucuronidase was performed to break down the conjugated forms and obtain more accurate exposure results. The most highly contaminated urine sample (containing $54.1 \mathrm{ng} / \mathrm{mL}$ of total TeA) found in our study was selected and used to evaluate the completeness of enzyme digestion. To $1 \mathrm{~mL}$ of the urine sample, $1 \mathrm{~mL}$ of enzyme solution containing different amounts of $\beta$-glucuronidase (200, $500,1000,2000$, and 5000 units) was added and incubated at $37^{\circ} \mathrm{C}$ overnight. The concentration of TeA was quantified, which increased along with the amount of $\beta$-glucuronidase and reached a platform at 1000 units. It indicated that 1000 units $\beta$-glucuronidase per $\mathrm{mL}$ urine was sufficient for the maximum release of TeA. Finally, 2000 units/mL urine was chosen in the study.

\subsection{Optimization of MS-MS Parameters}

For each analyte, more than three different multiple reaction monitoring (MRM) transitions were optimized via the injection of individual standard solutions. Key parameters that influence sensitivity for different MRM transitions were manually optimized, including declustering potential (DP) and collision energy (CE). To ensure sufficient sample ionization, a lower DP was selected to increase the abundance of the parent ion, and the total sensitivity increased correspondingly. For $\mathrm{AOH}, \mathrm{TeA}$, AME, and OT-alpha, the signal intensity was relatively higher in negative ion mode than in positive ion mode. Selection of product ions and optimization of CE were performed by individual infusions of each analyte standard. During the infusion, CE was tuned from 10-60 eV to produce the most intensive and stable product ion signal in real-time product-ion scan spectrum. Finally, two transitions with the greatest sensitivity and minimum impurity interference were selected for each compound. Each MS/MS transition and its corresponding optimal DP and CE are shown in Table 1. 
Table 1. MRM transitions and MS/MS parameters for 18 mycotoxin analytes.

\begin{tabular}{|c|c|c|c|c|c|}
\hline Compound & Precursor Ion & $\mathrm{Q} 1(m / z)$ & $\mathrm{Q} 3(m / z)$ & $\mathrm{CE}(\mathrm{eV})^{1}$ & $\mathrm{DP}(\mathrm{V})^{2}$ \\
\hline \multirow{2}{*}{ OTA } & \multirow{2}{*}[\mathrm{M}+\mathrm{H}]{$^{+}$} & \multirow{2}{*}{404.1} & $239.1^{\mathrm{a}}$ & 34 & 50 \\
\hline & & & 358.1 & 21 & 50 \\
\hline \multirow{2}{*}{ OTВ } & \multirow{2}{*}[\mathrm{M}+\mathrm{H}]{$^{+}$} & \multirow{2}{*}{371.1} & $205.9^{a}$ & 31 & 40 \\
\hline & & & 188.1 & 35 & 40 \\
\hline \multirow{2}{*}{ OT-alpha } & \multirow{2}{*}[\mathrm{M}-\mathrm{H}]{$^{-}$} & \multirow{2}{*}{255.0} & $211.0^{\mathrm{a}}$ & -21 & -30 \\
\hline & & & 167.1 & -32 & -30 \\
\hline \multirow{2}{*}{$\mathrm{FB}_{1}$} & \multirow{2}{*}[\mathrm{M}+\mathrm{H}]{$^{+}$} & \multirow{2}{*}{722.3} & $334.3^{\mathrm{a}}$ & 55 & 50 \\
\hline & & & 352.3 & 51 & 50 \\
\hline \multirow{2}{*}{$\mathrm{FB}_{2}$} & \multirow{2}{*}[\mathrm{M}+\mathrm{H}]{$^{+}$} & \multirow{2}{*}{706.4} & $336.1^{\mathrm{a}}$ & 50 & 50 \\
\hline & & & 354.2 & 46 & 50 \\
\hline \multirow{2}{*}{$\mathrm{FB}_{3}$} & \multirow{2}{*}[\mathrm{M}+\mathrm{H}]{$^{+}$} & \multirow{2}{*}{706.4} & $336.3^{\mathrm{a}}$ & 50 & 50 \\
\hline & & & 318.1 & 51 & 50 \\
\hline \multirow{2}{*}{$\mathrm{HFB}_{1}$} & \multirow{2}{*}[\mathrm{M}+\mathrm{H}]{$^{+}$} & 4063 & $334.1^{\mathrm{a}}$ & 35 & 40 \\
\hline & & 400.3 & 352.1 & 31 & 40 \\
\hline $\mathrm{HFB}_{2}$ & {$\left[\mathrm{M}+\mathrm{H}^{+}\right.$} & 390.3 & $336.2^{\mathrm{a}}$ & 33 & 35 \\
\hline $\mathrm{H}_{2}$ & {$[101+11]$} & 390.3 & 238.1 & 37 & 35 \\
\hline $\mathrm{AOH}$ & {$[\mathrm{M}-\mathrm{H}]^{-}$} & 257.0 & $215.0^{\mathrm{a}}$ & -34 & -130 \\
\hline AUI & {$[1 \mathrm{~V}-11]$} & & 147.0 & -43 & -130 \\
\hline & & & $214.0^{\mathrm{a}}$ & -30 & -100 \\
\hline ALI & {$[\mathrm{M}+\mathrm{H}]$} & 291.0 & 229.2 & -21 & -100 \\
\hline TEN & & & $312.1^{\mathrm{a}}$ & 29 & 110 \\
\hline IEN & {$[\mathrm{MI}+\mathrm{H}]$} & 415.3 & 256.0 & 40 & 110 \\
\hline TeA & {$\left[\mathrm{M}-\mathrm{H}^{-}\right.$} & 1961 & $139.0^{\mathrm{a}}$ & -25 & -50 \\
\hline IEA & {$[101-11]$} & 190.1 & 112.0 & -35 & -50 \\
\hline AME & {$[\mathrm{M}-\mathrm{H}]^{-}$} & 271.0 & $256.0^{\mathrm{a}}$ & -30 & -110 \\
\hline AIVIL & {$[101-11]$} & & 228.0 & -36 & -110 \\
\hline BEA & {$[\mathrm{M}+\mathrm{H}]^{+}$} & 784.5 & $244.2^{\mathrm{a}}$ & 41 & 240 \\
\hline & {$[1 \mathrm{N1}+1]$} & 184.5 & 134.3 & 92 & 240 \\
\hline ENNA & {$[\mathrm{M}+\mathrm{H}]^{+}$} & 6824 & $210.2^{\mathrm{a}}$ & 34 & 220 \\
\hline & {$\left[\begin{array}{lll}201 & 1\end{array}\right]$} & 002.4 & 228.2 & 38 & 220 \\
\hline & {$[\mathrm{M}+\mathrm{H}]^{+}$} & 6683 & $210.0^{\mathrm{a}}$ & 32 & 200 \\
\hline $\mathrm{ENNA}_{1}$ & {$[\mathrm{M}+\mathrm{H}]^{\prime}$} & 668.3 & 228.1 & 33 & 200 \\
\hline FNNB & {$\left[\mathrm{M}+\mathrm{Hl}^{+}\right.$} & 640.3 & $196.4^{\mathrm{a}}$ & 34 & 180 \\
\hline LIVIVD & {$[101+11]$} & $0+0.3$ & 241.2 & 33 & 180 \\
\hline $\mathrm{ENNB}_{1}$ & {$[\mathrm{M}+\mathrm{H}]^{+}$} & 654.3 & $196.0^{\mathrm{a}}$ & 33 & 180 \\
\hline & {$\left[\begin{array}{lll}101 & 11\end{array}\right]$} & - > & 210.2 & 35 & 180 \\
\hline${ }^{13} \mathrm{C}_{20}$-OTA & {$[\mathrm{M}+\mathrm{H}]^{+}$} & 424.2 & $250.1^{a}$ & 35 & 50 \\
\hline $20=01 \pi$ & {$[191+11]$} & $4 \angle 4.2$ & 337.1 & 20 & 50 \\
\hline${ }^{13} \mathrm{C}_{34}-\mathrm{FB}_{1}$ & {$[\mathrm{M}+\mathrm{H}]^{+}$} & 7567 & $374.4^{\mathrm{a}}$ & 53 & 50 \\
\hline & & 700.7 & 356.3 & 55 & 50 \\
\hline${ }^{13} \mathrm{C}_{34}-\mathrm{FB}_{2}$ & {$[\mathrm{M}+\mathrm{H}]^{+}$} & 7407 & $358.3^{\mathrm{a}}$ & 50 & 50 \\
\hline & & 140.7 & 376.2 & 47 & 50 \\
\hline${ }^{13} \mathrm{C}_{34}-\mathrm{FB}_{3}$ & {$[\mathrm{M}+\mathrm{H}]^{+}$} & 740.7 & $358.2^{\mathrm{a}}$ & 50 & 50 \\
\hline & & & 376.1 & 47 & 50 \\
\hline TeA-d ${ }_{13}$ & {$[\mathrm{M}-\mathrm{H}]^{-}$} & 210.3 & $143.0^{\mathrm{a}}$ & -27 & -50 \\
\hline 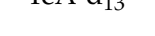 & 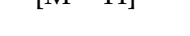 & & 115.0 & -34 & -50 \\
\hline TEN-d $\mathrm{d}_{3}$ & {$[\mathrm{M}+\mathrm{H}]^{+}$} & 418.2 & $314.9^{\mathrm{a}}$ & 30 & 140 \\
\hline & 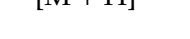 & 410.2 & 305.4 & 19 & 140 \\
\hline AME- $d_{3}$ & {$[\mathrm{M}-\mathrm{H}]^{-}$} & 274.0 & $256.0^{\mathrm{a}}$ & -32 & -110 \\
\hline & & 2.1 .0 & 228.1 & -39 & -110 \\
\hline & {$[\mathrm{M}+\mathrm{H}]^{+}$} & 2589 & $215.1^{\mathrm{a}}$ & -31 & -130 \\
\hline $\mathrm{A} \cup \Pi-\mathrm{a}_{2}$ & {$[111+11]$} & 200.9 & 148.0 & -43 & -130 \\
\hline
\end{tabular}

${ }^{\mathrm{a}}$ Quantification ion; ${ }^{1} \mathrm{CE}$, collision energy $(\mathrm{eV}) ;{ }^{2} \mathrm{DP}$, declustering potential (V).

The ion source parameters, including ionization mode, curtain gas (CUR), collision gas (CAD), ion spray voltage (IS), temperature (TEM), ion source gas 1 (GS1), and ion source gas 2 (GS2), were manually optimized for better sensitivity $(\mathrm{S} / \mathrm{N})$ for most compounds, and the results are summarized in Table 2. 
Table 2. Ion source parameters for mycotoxin biomarker analysis.

\begin{tabular}{cc}
\hline Ion Source Parameters & Settings \\
\hline Curtain gas CUR (psi) & 20 \\
Collision Gas CAD & 10 \\
Ion Spray Voltage (IS) $(\mathrm{V})$ & $5500 /-4500$ \\
Temperature (TEM) $\left({ }^{\circ} \mathrm{C}\right)$ & 600 \\
Ion Source Gas 1 (GS1) $(\mathrm{psi})$ & 65 \\
Ion Source Gas 2 (GS2) $(\mathrm{psi})$ & 55 \\
\hline
\end{tabular}

\subsection{Optimization of Chromatographic Separation}

Chromatographic separation is the next equally crucial part in a reliable UHPLC-MS/MS method. Chromatographic performance was optimized, including the column, mobile phase and its additives, flow rate, elution gradient, and proper column temperature. The CORTECS C18 UPLC column was selected due to its $1.6 \mu \mathrm{m}$ core-shell packing particles that provide a more effective separation of the analytes in a shorter run-time, compared to a porous column (BEH C18 UPLC column). After the mobile phase was optimized (Figure 4), the 18 analytes were divided into two groups for instrument analysis. For the first group of compounds $\left(\mathrm{OTA}, \mathrm{OTB}, \mathrm{FB}_{1}, \mathrm{FB}_{2}, \mathrm{FB}_{3}, \mathrm{HFB}_{1}\right.$, and $\left.\mathrm{HFB}_{2}\right)$, acetonitrile gave a lower background signal and stronger elution ability than methanol. a ratio of 1:1 methanol $(\mathrm{MeOH})$ to acetonitrile (ACN) was chosen as solvent $\mathrm{B}$, for optimal separation efficiency and sensitivity. As shown in Figure 4a, an acidic mobile phase is necessary for most ochratoxins and fumonisins, since $\mathrm{H}^{+}$can improve their ionization efficiency. More importantly, satisfactory chromatographic separation of fumonisins can easily be achieved in acid conditions. Even though, different proportions of aqueous formic acid (FAc) had little effect on the sensitivity. $\mathrm{HFB}_{1}$ and $\mathrm{HFB}_{2}$ had larger peak areas in neutral condition. However, they displayed tailing peaks in neutral and alkaline conditions, while gave sharp peaks in acidic mobile phase. As a consequence, HFB1 and HFB2 were classified into the first group using $0.2 \%$ FAc and MeOH:ACN (1:1, v:v) as an optimal mobile phase. Seven analytes achieved satisfactory separation, as displayed in Figure 5a. For the second group (OT-alpha, Alternaria toxins, ENNs, and BEA), acetonitrile was used as solvent B. Ammonium acetate, ammonium formate, and ammonium hydroxide at different concentrations were evaluated as additives in the aqueous phase. TeA, OT-alpha, ALT, AOH, and AME had the largest peak areas in water with no additives, but obvious peak broadening and tailing were observed. As a result, the aqueous phase contained $5 \mathrm{mmol} / \mathrm{L}$ ammonium acetate and $0.01 \%$ ammonia was used as the optimal solvent a for the second group, after comprehensive consideration of peak shape and mass signal intensity. The results are shown in Figure $4 \mathrm{~b}$. The representative chromatogram of the second group is presented in Figure $5 \mathrm{~b}$. 

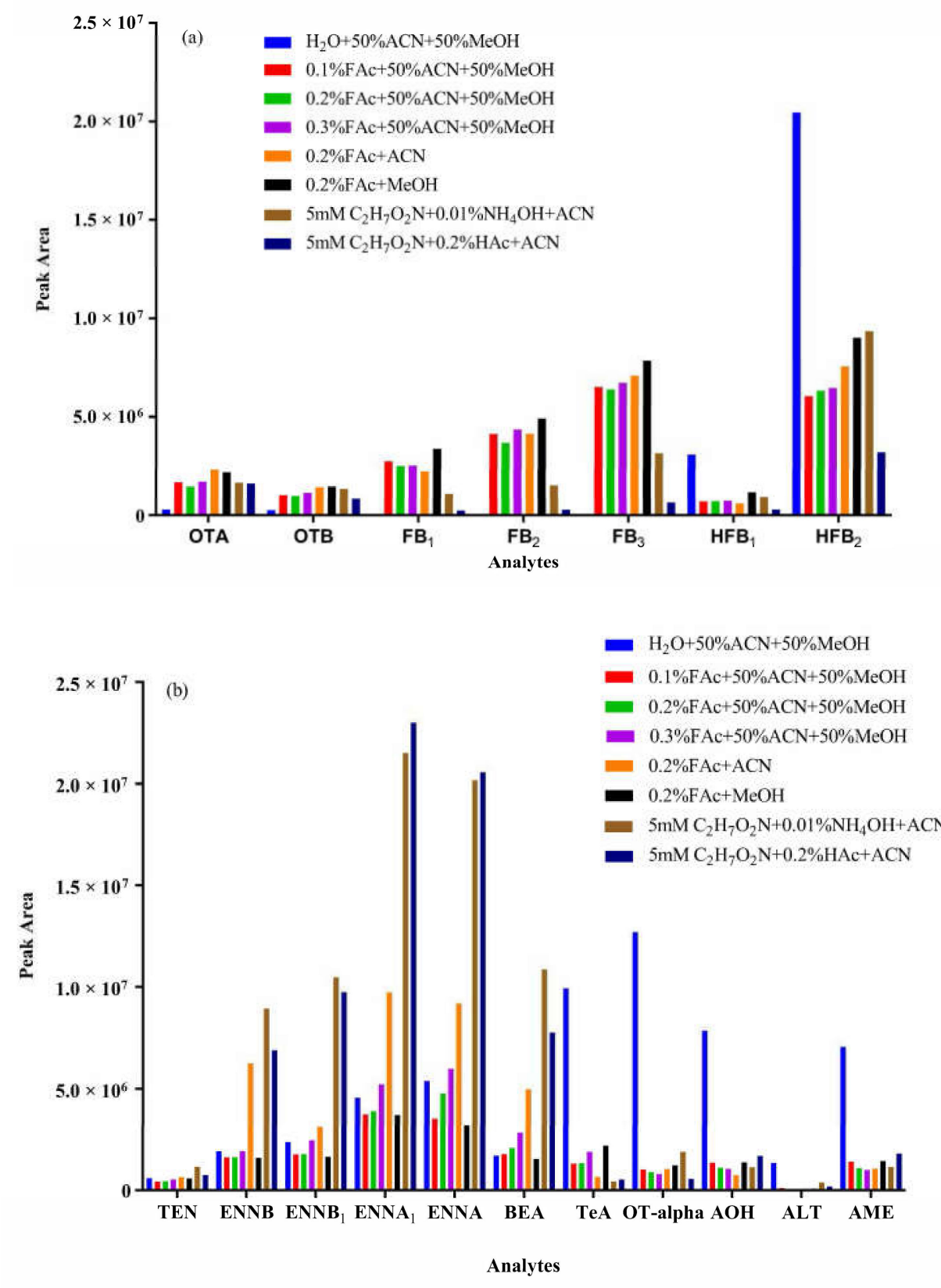

Figure 4. Evaluation of the effects of additives in the mobile phase on the peak areas for (a) 7 analytes (OTA, OTB, FB $1, \mathrm{FB}_{2}, \mathrm{FB}_{3}, \mathrm{HFB}_{1}$, and $\mathrm{HFB}_{2}$ ) and (b) 11 analytes (OT-alpha, ATs, ENNs, and BEA). Abbreviations: FAc, formic acid; $\mathrm{HAc}$, acetic acid; $\mathrm{C}_{2} \mathrm{H}_{7} \mathrm{O}_{2} \mathrm{~N}$, ammonium acetate; $\mathrm{NH}_{4} \mathrm{OH}$, ammonia water solution; $\mathrm{ACN}$, acetonitrile; $\mathrm{MeOH}$, methanol. 

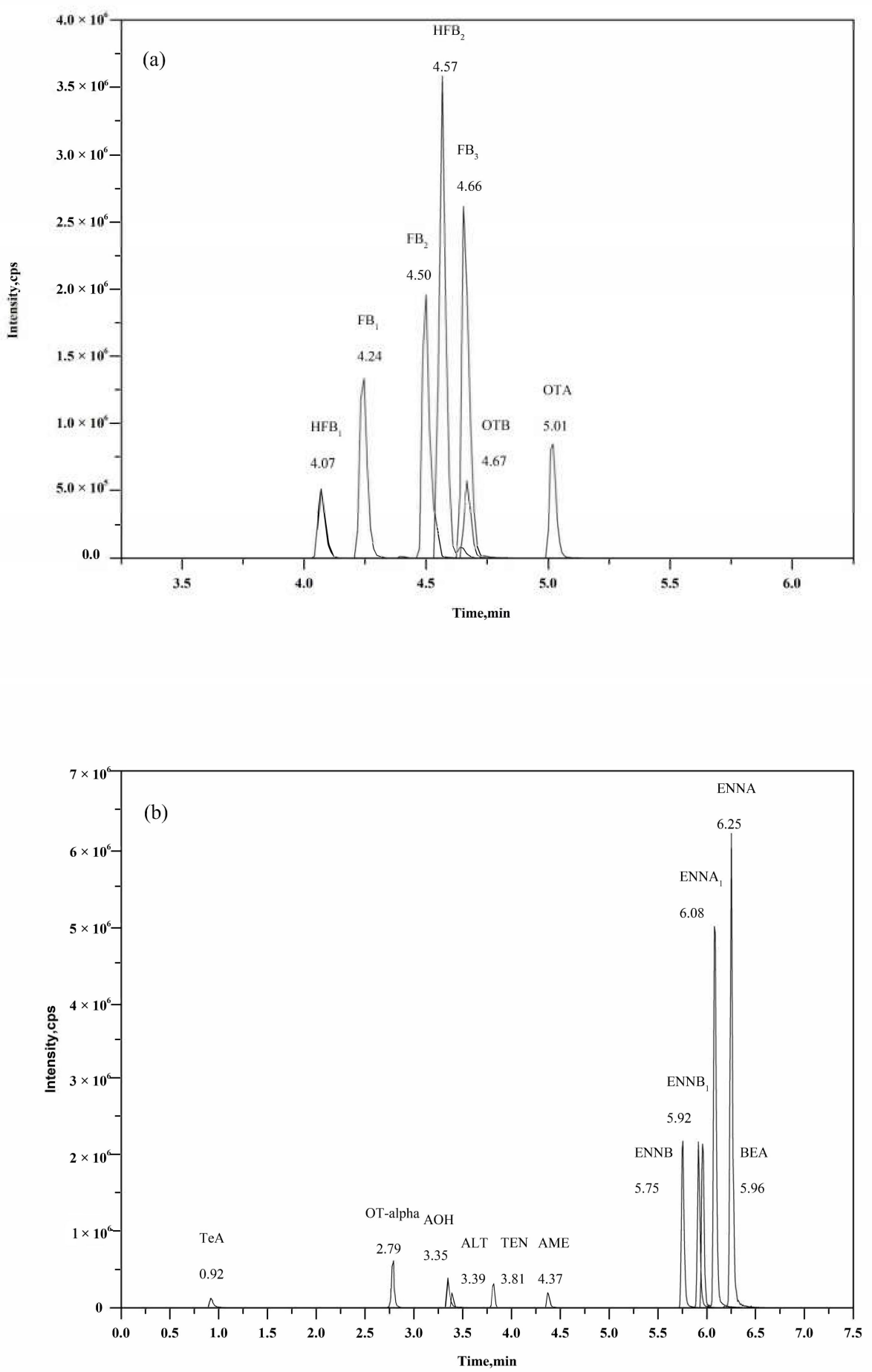

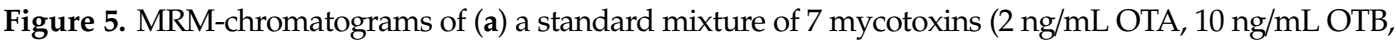
$10 \mathrm{ng} / \mathrm{mL} \mathrm{FB}_{1}, 10 \mathrm{ng} / \mathrm{mL} \mathrm{FB}_{2}, 10 \mathrm{ng} / \mathrm{mL} \mathrm{FB}_{3}, 5 \mathrm{ng} / \mathrm{mL} \mathrm{HFB}_{1}$, and $\left.10 \mathrm{ng} / \mathrm{mL} \mathrm{HFB}_{2}\right)$ and $(\mathbf{b})$ a standard mixture of 11 mycotoxins ( $5 \mathrm{ng} / \mathrm{mL}$ OT-alpha, $10 \mathrm{ng} / \mathrm{mL}$ AOH, $20 \mathrm{ng} / \mathrm{mL}$ TeA, $20 \mathrm{ng} / \mathrm{mL}$ ALT, $2 \mathrm{ng} / \mathrm{mL}$ AME, $2 \mathrm{ng} / \mathrm{mL}$ TEN, 1 ng/mL ENNB, 1 ng/mL ENNB 1 , 2 ng/mL ENNA, 2 ng/mL ENNA 1 , and 2 ng/mL BEA).

\subsection{Validation Experiments}

The linearity, method recovery, intra-day and inter-day precision, limit of detection (LOD), and limit of quantification (LOQ) were evaluated for the established method (involving enzyme hydrolysis, SPE purification, and UHPLC-MS/MS analysis), following the guidelines of Commission Decision 200/657/EC [46], EMEA [47], and FDA [48]. 
Calibration curves prepared in pure solvent (ACN/0.2\%Fac, 50/50) were linear from LOQ up to $25 \mathrm{ng} / \mathrm{mL}$ for ENNB and ENNB $1,50 \mathrm{ng} / \mathrm{mL}$ for ENNA, ENNA 1 , TEN, AME, and OTA, $125 \mathrm{ng} / \mathrm{mL}$ for $\mathrm{HFB}_{1}$ and OT-alpha, $250 \mathrm{ng} / \mathrm{mL}$ for $\mathrm{FB}_{1}, \mathrm{FB}_{2}, \mathrm{FB}_{3}, \mathrm{HFB}_{2}$, and $\mathrm{AOH}$, and $500 \mathrm{ng} / \mathrm{mL}$ for the remaining mycotoxins with $1 / \mathrm{x}^{2}$ weighting (Table 3 ). Linearity was assessed on three consecutive days, with the average correlation coefficients in range of 0.997 to 0.999 , which demonstrated good linear responses for all analytes.

Table 3. Sensitivity, extraction recovery, matrix effect and linearity of each analyte.

\begin{tabular}{|c|c|c|c|c|c|c|c|}
\hline Analyte & $\begin{array}{l}R_{E} \\
(\%)\end{array}$ & $\begin{array}{l}\text { SSE } \\
(\%)\end{array}$ & $\begin{array}{l}\mathrm{R}_{\mathrm{A}} \\
(\%)\end{array}$ & $\begin{array}{c}\text { Linear Range }{ }^{1} \\
(\mathrm{ng} / \mathrm{mL})\end{array}$ & $\mathbf{R}^{2}$ & $\begin{array}{c}\text { LOD }^{2} \\
(\mathrm{ng} / \mathrm{mL})\end{array}$ & $\begin{array}{c}\mathrm{LOQ}^{3} \\
(\mathrm{ng} / \mathrm{mL})\end{array}$ \\
\hline OTA & $107 \%$ & $49.1 \%$ & $52.3 \%$ & $0.05-50$ & 0.999 & 0.01 & 0.02 \\
\hline ОТВ & $91.6 \%$ & $64.3 \%$ & $58.9 \%$ & $0.25-250$ & 0.998 & 0.01 & 0.04 \\
\hline $\mathrm{FB}_{1}$ & $93.7 \%$ & $77.3 \%$ & $72.4 \%$ & $0.25-250$ & 0.998 & 0.02 & 0.05 \\
\hline $\mathrm{FB}_{2}$ & $93.7 \%$ & $69.3 \%$ & $64.9 \%$ & $0.25-250$ & 0.999 & 0.02 & 0.05 \\
\hline $\mathrm{FB}_{3}$ & $93.5 \%$ & $95.7 \%$ & $89.5 \%$ & $0.25-250$ & 0.998 & 0.01 & 0.03 \\
\hline $\mathrm{HFB}_{1}$ & $104 \%$ & $64.2 \%$ & $66.6 \%$ & $0.25-125$ & 0.998 & 0.01 & 0.02 \\
\hline $\mathrm{HFB}_{2}$ & $111 \%$ & $56.9 \%$ & $63.0 \%$ & $0.25-250$ & 0.997 & 0.02 & 0.04 \\
\hline $\mathrm{TeA}$ & $87.3 \%$ & $41.8 \%$ & $36.4 \%$ & $0.5-500$ & 0.999 & 0.2 & 0.5 \\
\hline OT-alpha & $99.0 \%$ & $12.2 \%$ & $12.1 \%$ & $0.25-125$ & 0.998 & 0.02 & 0.05 \\
\hline $\mathrm{AOH}$ & $75.2 \%$ & $16.4 \%$ & $12.4 \%$ & $0.25-250$ & 0.998 & 0.01 & 0.04 \\
\hline ALT & $85.2 \%$ & $93.4 \%$ & $79.6 \%$ & $0.5-500$ & 0.998 & 0.02 & 0.07 \\
\hline AME & $74.6 \%$ & $120 \%$ & $89.3 \%$ & $0.01-50$ & 0.999 & 0.001 & 0.003 \\
\hline TEN & $88.4 \%$ & $49.7 \%$ & $43.9 \%$ & $0.05-50$ & 0.998 & 0.002 & 0.01 \\
\hline ENNB & $90.0 \%$ & $101 \%$ & $90.8 \%$ & $0.001-25$ & 0.998 & 0.0001 & 0.0002 \\
\hline $\mathrm{ENNB}_{1}$ & $84.1 \%$ & $104 \%$ & $87.1 \%$ & $0.001-25$ & 0.997 & 0.0001 & 0.0002 \\
\hline ENNA & $71.6 \%$ & $101 \%$ & $72.5 \%$ & $0.005-50$ & 0.997 & 0.0002 & 0.0008 \\
\hline ENNA $_{1}$ & $77.5 \%$ & $101 \%$ & $78.5 \%$ & $0.005-50$ & 0.998 & 0.0001 & 0.0005 \\
\hline BEA & $85.5 \%$ & $89.7 \%$ & $76.6 \%$ & $0.005-50$ & 0.998 & 0.0002 & 0.0006 \\
\hline
\end{tabular}

${ }^{1}$ Linear range of calibration curves prepared in pure solvent. ${ }^{2}$ Limit of detection in urine. ${ }^{3}$ Limit of quantification in urine.

Method recovery $\left(\mathrm{R}_{\mathrm{M}}\right)$ and precision values were determined from spiked urine samples at three (low, medium, and high) levels with internal standards correction, as described in the Section 4 . Nearly all the analytes displayed acceptable recoveries with internal standard correction, ranging from $74.0 \%$ to $133 \%$. The intra-day precision and inter-day precision were $0.5 \%-8.7 \%$ and $2.4 \%-13.4 \%$, respectively. The recovery and precision data for all concentrations are shown in Table 4. 
Table 4. Recovery and precision of the developed method in spiked urine samples $(n=6)$.

\begin{tabular}{|c|c|c|c|c|c|}
\hline \multirow{2}{*}{ Analyte } & \multirow{2}{*}{$\begin{array}{l}\text { Spiked Level } \\
\text { in Urine } \\
(\mathrm{ng} / \mathrm{mL})\end{array}$} & \multirow{2}{*}{$\begin{array}{c}\text { Measured } \\
\text { Value in Urine } \\
(\mathrm{ng} / \mathrm{mL})\end{array}$} & \multirow{2}{*}{$\begin{array}{l}\text { Method } \\
\text { Recovery } \\
(\%)\end{array}$} & \multicolumn{2}{|c|}{ RSD (\%) } \\
\hline & & & & $\begin{array}{c}\text { Intra-day } \\
(n=6)\end{array}$ & $\begin{array}{c}\text { Inter-day } \\
(n=18)\end{array}$ \\
\hline \multirow{3}{*}{ OTA } & 0.1 & 0.10 & 100 & 2.8 & 4.8 \\
\hline & 1 & 1.11 & 111 & 3.3 & 3.5 \\
\hline & 4 & 4.15 & 104 & 2.4 & 3.8 \\
\hline \multirow{3}{*}{ ОТВ } & 0.5 & 0.41 & 82.1 & 1.8 & 10.8 \\
\hline & 5 & 4.18 & 83.5 & 2.8 & 4.6 \\
\hline & 20 & 17.4 & 86.9 & 0.9 & 5.8 \\
\hline \multirow{3}{*}{$\mathrm{FB}_{1}$} & 0.5 & 0.47 & 94.6 & 3.1 & 3.8 \\
\hline & 5 & 4.95 & 99.0 & 3.6 & 4.9 \\
\hline & 20 & 19.5 & 97.4 & 2.5 & 3.4 \\
\hline \multirow{3}{*}{$\mathrm{FB}_{2}$} & 0.5 & 0.49 & 98.0 & 3.7 & 4.8 \\
\hline & 5 & 5.28 & 106 & 3.8 & 10.0 \\
\hline & 20 & 18.3 & 91.7 & 3.5 & 6.5 \\
\hline \multirow{3}{*}{$\mathrm{FB}_{3}$} & 0.5 & 0.47 & 93.3 & 2.7 & 4.3 \\
\hline & 5 & 5.20 & 104 & 4.6 & 6.3 \\
\hline & 20 & 19.4 & 97.1 & 2.5 & 6.0 \\
\hline \multirow{3}{*}{$\mathrm{HFB}_{1}$} & 0.25 & 0.21 & 83.5 & 0.9 & 3.6 \\
\hline & 2.5 & 2.25 & 89.8 & 0.5 & 5.1 \\
\hline & 10 & 8.83 & 88.3 & 3.5 & 5.6 \\
\hline \multirow{4}{*}{$\mathrm{HFB}_{2}$} & 0.5 & 0.37 & 74.0 & 3.1 & 9.5 \\
\hline & 5 & 4.22 & 84.5 & 1.1 & 3.5 \\
\hline & 20 & 16.4 & 82.0 & 0.6 & 6.6 \\
\hline & 1 & 0.93 & 93.0 & 6.9 & 9.2 \\
\hline \multirow[t]{2}{*}{$\mathrm{TeA}$} & 10 & 9.84 & 98.4 & 6.2 & 8.3 \\
\hline & 40 & 38.3 & 95.8 & 1.3 & 4.4 \\
\hline \multirow{3}{*}{ OT-alpha } & 0.25 & 0.21 & 84.4 & 1.2 & 8.7 \\
\hline & 2.5 & 2.00 & 79.9 & 4.1 & 6.5 \\
\hline & 10 & 0.88 & 88.1 & 1.9 & 8.7 \\
\hline \multirow{4}{*}{$\mathrm{AOH}$} & 0.5 & 0.538 & 108 & 1.4 & 7.5 \\
\hline & 5 & 5.33 & 107 & 2.2 & 4.4 \\
\hline & 20 & 21.3 & 107 & 2.2 & 2.7 \\
\hline & 1 & 1.15 & 115 & 8.7 & 13.4 \\
\hline \multirow[t]{3}{*}{ ALT } & 10 & 13.3 & 133 & 6.3 & 11.1 \\
\hline & 40 & 51.8 & 130 & 3.2 & 7.3 \\
\hline & 0.1 & 0.08 & 80.9 & 2.7 & 5.2 \\
\hline \multirow[t]{3}{*}{ AME } & 1 & 0.92 & 92.2 & 0.7 & 2.6 \\
\hline & 4 & 3.81 & 95.2 & 1.7 & 2.4 \\
\hline & 0.1 & 0.11 & 110 & 4.8 & 6.0 \\
\hline \multirow[t]{3}{*}{ TEN } & 1 & 1.08 & 108 & 2.7 & 2.8 \\
\hline & 4 & 4.12 & 103 & 1.2 & 3.2 \\
\hline & 0.05 & 0.05 & 94.6 & 1.7 & 4.9 \\
\hline ENNB & 0.5 & 0.49 & 98.5 & 2.8 & 4.9 \\
\hline & 2 & 2.02 & 101 & 2.7 & 4.3 \\
\hline & 0.05 & 0.05 & 95.0 & 6.9 & 7.2 \\
\hline $\mathrm{ENNB}_{1}$ & 0.5 & 0.49 & 97.4 & 1.1 & 5.9 \\
\hline & 2 & 1.92 & 96.0 & 3.2 & 6.2 \\
\hline & 0.1 & 0.09 & 84.3 & 1.7 & 8.2 \\
\hline ENNA & 1 & 0.90 & 89.9 & 3.6 & 7.0 \\
\hline & 4 & 3.79 & 94.7 & 3.2 & 6.9 \\
\hline & 0.1 & 0.09 & 90.0 & 2.9 & 9.9 \\
\hline $\mathrm{ENNA}_{1}$ & 1 & 1.00 & 99.4 & 1.4 & 7.5 \\
\hline & 4 & 3.90 & 97.5 & 3.8 & 7.5 \\
\hline & 0.1 & 0.08 & 80.8 & 4.1 & 5.8 \\
\hline BEA & 1 & 0.77 & 76.4 & 1.8 & 3.9 \\
\hline & 4 & 3.20 & 80.1 & 2.7 & 4.3 \\
\hline
\end{tabular}

LOD and LOQ of each analyte in urine was evaluated by spiking urine matrix at low concentration levels that generated signal-to-noise ratios $(\mathrm{S} / \mathrm{N})$ of 3 and 10, respectively. The LOQ ranged from ppt levels $(0.2 \mathrm{pg} / \mathrm{mL}$ for ENNB) to ppb levels $(0.5 \mathrm{ng} / \mathrm{mL}$ for TeA). The other detailed information has been listed in Table 3. This method showed improved LOD and LOQ compared to published methods for the same compounds in urine, which is summarized in Table 5. 
Table 5. Analytical methods for the determination of mycotoxins in urine.

\begin{tabular}{|c|c|c|c|c|}
\hline Analyte & Sample Preparation & Analysis Method & $\begin{array}{c}\mathrm{LOQ} \\
(\mu \mathrm{g} / \mathrm{L})\end{array}$ & Reference \\
\hline $\begin{array}{c}\text { OTA, OT-alpha, FB } 1, \mathrm{FB}_{2}, \mathrm{FB}_{3} \\
\mathrm{HFB}_{1}, \mathrm{AOH}, \mathrm{AME}\end{array}$ & QuEChERS & UPLC-MS/MS & $0.02-1.0$ & [15] \\
\hline OTA, OT-alpha, $\mathrm{FB}_{1}$, ENNB & Dilute and shoot & LC-MS/MS & $0.0005-0.1$ & [43] \\
\hline $\mathrm{OTA}, \mathrm{FB}_{1}, \mathrm{AOH}$ & SPE & UHPLC-MS/MS & $0.001-0.03$ & [3] \\
\hline OTA, $\mathrm{FB}_{1}$ & LLE+ QuEChERS & UPLC-MS/MS & $0.16-1.1$ & [36] \\
\hline OTA, OT-alpha, FB 1 & Direct method & UHPLC-MS/MS & $0.1-0.5$ & [8] \\
\hline OTA, OT-alpha & LLE & HPLC-FD & 0.02 & [32] \\
\hline OTA, OT-alpha, $\mathrm{FB}_{1}, \mathrm{FB}_{2}, \mathrm{FB}_{3}$ & Direct method/ICA & LC-MS/MS & $0.003-0.2$ & [41] \\
\hline OTA, $\mathrm{FB}_{1}, \mathrm{FB}_{2}$ & Dilute and shoot & LC-MS/MS & $0.017-0.17$ & [4] \\
\hline OTA, $\mathrm{FB}_{1}, \mathrm{FB}_{2}$ & $\mathrm{SPE}+\mathrm{SPE}$ & LC-MS/MS & $0.007-0.017$ & [19] \\
\hline ENNB & $\begin{array}{c}\text { salting-out liquid-liquid } \\
\text { extraction }\end{array}$ & $\begin{array}{c}\text { UHPLC-Q-Orbitrap } \\
\text { HRMS }\end{array}$ & 0.001 & [38] \\
\hline $\mathrm{ENN}_{\mathrm{S}}, \mathrm{BEA}$ & SPE & LC-MS/MS & $0.005-0.02$ & [7] \\
\hline OTA, OT-alpha, $\mathrm{FB}_{1}, \mathrm{HFB}_{1}$ & $\mathrm{LLE}+\mathrm{SPE}$ & LC-MS/MS & $0.06-1.02$ & [14] \\
\hline
\end{tabular}

Matrix effects (SSE), extraction recovery $\left(\mathrm{R}_{\mathrm{E}}\right)$, and apparent recovery $\left(\mathrm{R}_{\mathrm{A}}\right)$ were also determined to evaluate the sample preparation step (including enzyme hydrolysis) and instrumental detection step separately. It was performed using three sets of calibration curves without internal standard correction as described in the Section 4. As shown in Table 3, due to the complexity of the matrix, the SSE ranged from $12.2 \%$ to $119.6 \%$. There was severe signal suppression for OT-alpha and $A O H$. $R_{E}$ values were satisfactory for all the analytes in a range of $71.6 \% \sim 111 \%$. The results demonstrated an effective analyte extraction, while showing the necessity of internal standard compensation. Based on the results, internal standards with similar $\mathrm{R}_{\mathrm{A}}$ were selected as reference internal standards for compounds lacking commercial internal standards. ${ }^{13} \mathrm{C}-\mathrm{FB}_{2}$ was used as the reference internal standard for $\mathrm{HFB}_{1}$, $\mathrm{HFB}_{2}$, and OTB quantification. AME- $\mathrm{d}_{3}$ was selected for ENNs and BEA quantification. ${ }^{13} \mathrm{C}_{-} \mathrm{FB}_{1}$ and $\mathrm{AOH}-\mathrm{d}_{2}$ were employed to more accurately quantitate ALT and OT-alpha after evaluation, respectively. The $\mathrm{R}_{\mathrm{E}}$ was $71.6 \%-110.8 \%$, indicating great extraction efficiency of sample preparation.

\subsection{Application of the Method}

Sixty samples of human urine were analyzed in duplicate using the optimized method. QC samples were included in each batch of analysis, and their measured values should be within $\pm 15 \%$ of the theoretical values. After $\beta$-glucuronidase digestion, the results revealed that $98.3 \%$ of the analyzed samples contained at least one mycotoxin, and 35 urine samples $(58.3 \%)$ contained four or more mycotoxins. Overall, Alternaria mycotoxins were highly detected followed by ochratoxins. The most frequently detected was TeA $(86.7 \%)$, with concentrations of $4.4 \pm 8.7 \mathrm{ng} / \mathrm{mL}$. AME had the second highest detection rate at $83.8 \%$ of samples, with concentrations ranging from $<\mathrm{LOD}$ to $0.167 \mathrm{ng} / \mathrm{mL}$. Similar detection rates of TEN (38.3\%) and AOH (28.3\%) were observed. OTA and OT-alpha were found in about one-third of the samples with the mean concentrations of $0.02 \pm 0.02$ and $0.22 \pm 0.42 \mathrm{ng} / \mathrm{mL}$, respectively. ENNB $(40.0 \%)$ were the most frequently detected Fusarium toxins. $\mathrm{FB}_{1}(3.3 \%), \mathrm{FB}_{3}(1.7 \%)$, and $\mathrm{HFB}_{1}(1.7 \%)$ were rarely detected. The remaining ENNs, $\mathrm{OTB}, \mathrm{FB}_{2}, \mathrm{HFB}_{2}$, and ALT were not detected in any of the analyzed samples. The relative intensities of two product ions and the retention time (RT) were used to identify each compound, according to the Commission Decision 2002/657/EC. The results of all samples are summarized in Table 6, demonstrating sufficient sensitivity and applicability of this method for human biomonitoring. Several representative MRM-chromatograms of naturally contaminated samples are displayed in Figure 6. 
Table 6. Occurrence of mycotoxin biomarkers in urine samples with enzyme treatment $(n=60)$.

\begin{tabular}{ccccc}
\hline \multirow{2}{*}{$\begin{array}{c}\text { Mycotoxin } \\
\text { Biomarkers }\end{array}$} & \multicolumn{4}{c}{ Total Concentrations } \\
\cline { 2 - 5 } & Positive (\%) & $\begin{array}{c}\text { Mean } \pm \text { SD } \\
\mathbf{( n g} / \mathbf{m L})\end{array}$ & $\begin{array}{c}\text { Range } \\
\mathbf{( n g} / \mathbf{m L})\end{array}$ & $\begin{array}{c}\text { Median } \\
\mathbf{( n g} / \mathbf{m L})\end{array}$ \\
\hline OTA & $24(40 \%)$ & $0.02 \pm 0.02$ & $<$ LOD 0.14 & 0.005 \\
OT-alpha & $20(33.3 \%)$ & $0.22 \pm 0.42$ & $<$ LOD 2.38 & 0.001 \\
$\mathrm{FB}_{1}$ & $2(3.3 \%)$ & $0.01 \pm 0.01$ & $<$ LOD 0.07 & 0.01 \\
$\mathrm{FB}_{3}$ & $1(1.7 \%)$ & 0.01 & $<$ LOD $\sim 0.01$ & 0.005 \\
$\mathrm{HFB}_{1}$ & $1(1.7 \%)$ & $0.01 \pm 0.01$ & $<$ LOD 0.09 & 0.005 \\
$\mathrm{TeA}$ & $52(86.7 \%)$ & $4.4 \pm 8.7$ & $<$ LOD 54.1 & 1.4 \\
$\mathrm{AOH}$ & $17(28.3 \%)$ & $0.34 \pm 1.09$ & $<$ LOD 7.68 & 0.01 \\
$\mathrm{AME}$ & $50(83.3 \%)$ & $0.059 \pm 0.049$ & $<$ LOD 0.167 & 0.048 \\
$\mathrm{TEN}$ & $23(38.3 \%)$ & $0.024 \pm 0.042$ & $<$ LOD 0.193 & 0.001 \\
ENNB & $24(40.0 \%)$ & $0.0002 \pm 0.0002$ & $<$ LOD 0.0014 & 0.0001 \\
\hline
\end{tabular}

For calculation of mean, standard deviation and median values, concentration $<$ LOD was assigned half the LOD.

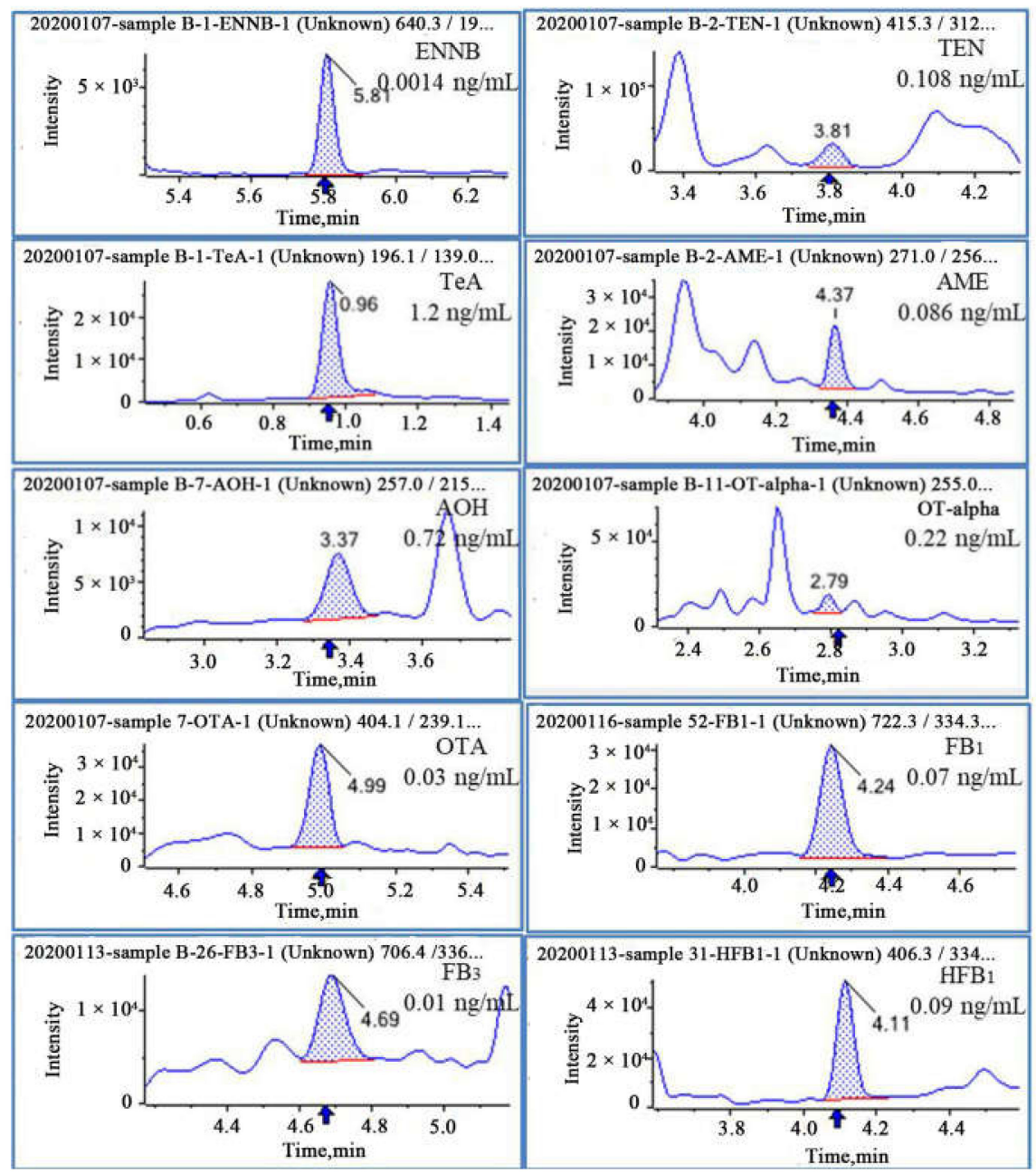

Figure 6. Representative MRM-chromatograms of urine samples with natural contamination of mycotoxins. ENNB, TEN, TeA, $\mathrm{AME}, \mathrm{AOH}, \mathrm{FB}_{1}, \mathrm{FB}_{3}, \mathrm{HFB}_{1}$, OT-alpha, and OTA were found.

\section{Conclusions}

This study reports on the development of an accurate and sensitive UHPLC-MS/MS method for the determination of 18 mycotoxins in human urine. The recoveries of target analytes ranged from $74.0 \%$ to $133 \%$, with inter-day RSD being less than $13.4 \%$. These values are within the acceptable range. After optimization, the LOQs were in the range of 0.0002 to $0.5 \mathrm{ng} / \mathrm{mL}$, exhibiting good 
sensitivity. The method was optimized and successfully applied to human urine analysis. Ten of the 18 biomarkers were detected, and at least one biomarker was found in all but one of the samples. In summation, the optimized analytical strategy represents a reliable tool for mycotoxin exposure assessment and contributes to more relevant studies. In the analysis of 60 urine samples, the high occurrence and concentration levels of TeA, AME, and ochratoxins are worthy of attention in future risk assessment studies.

\section{Materials and Methods}

\subsection{Reagents and Chemicals}

The following mycotoxin and isotopically-labeled mycotoxin standard solutions were purchased from Romer labs (Tulln, Lower Austria, Austria): OTA, OTB, OT-alpha, $\mathrm{FB}_{1}, \mathrm{FB}_{2}, \mathrm{FB}_{3}, \mathrm{HFB}_{1}, \mathrm{AOH}, \mathrm{AME}$, ALT, TEN, TeA, BEA, ENNA, ENNA 1 , ENNB, ENNB $1,{ }^{13} \mathrm{C}_{20}$-OTA, ${ }^{13} \mathrm{C}_{34}-\mathrm{FB}_{1},{ }^{13} \mathrm{C}_{34}-\mathrm{FB}_{2}$, and ${ }^{13} \mathrm{C}_{34}-\mathrm{FB}_{3}$. Solid powder of the $\mathrm{HFB}_{2}$ standard, as well as TEN-d $\mathrm{d}_{3}, \mathrm{AOH}-\mathrm{d}_{2}, \mathrm{TeA}-\mathrm{d}_{13}$, and AME- $\mathrm{d}_{3}$, were obtained from Toronto Research Chemicals (TRC, Toronto, ONT, Canada). Solid standard substances were dissolved in pure acetonitrile (ACN) or ACN/water (50/50). Individual standard solutions were kept at $-30{ }^{\circ} \mathrm{C}$ According to the different sensitivities of each analyte, a multi-standard stock solution was prepared in ACN/water (50/50) containing OTA $(0.5 \mu \mathrm{g} / \mathrm{mL}), \mathrm{HFB}_{1}(1.25 \mu \mathrm{g} / \mathrm{mL})$, OTB $(2.5 \mu \mathrm{g} / \mathrm{mL})$, $\mathrm{FB}_{1}(2.5 \mu \mathrm{g} / \mathrm{mL}), \mathrm{FB}_{2}(2.5 \mu \mathrm{g} / \mathrm{mL}), \mathrm{FB}_{3}(2.5 \mu \mathrm{g} / \mathrm{mL})$, and $\mathrm{HFB}_{2}(2.5 \mu \mathrm{g} / \mathrm{mL})$. a second multi-standard stock solution was prepared in pure ACN containing ENNB $(0.25 \mu \mathrm{g} / \mathrm{mL})$, ENNB $_{1}(0.25 \mu \mathrm{g} / \mathrm{mL})$, ENNA $(0.5 \mu \mathrm{g} / \mathrm{mL})$, ENNA $_{1}(0.5 \mu \mathrm{g} / \mathrm{mL})$, BEA $(0.5 \mu \mathrm{g} / \mathrm{mL})$, TEN $(0.5 \mu \mathrm{g} / \mathrm{mL})$, AME $(0.5 \mu \mathrm{g} / \mathrm{mL})$, OT-alpha $(1.25 \mu \mathrm{g} / \mathrm{mL}), \mathrm{AOH}(2.5 \mu \mathrm{g} / \mathrm{mL})$, ALT $(5 \mu \mathrm{g} / \mathrm{mL})$, and TeA $(5 \mu \mathrm{g} / \mathrm{mL})$. Both solutions were stored at $-30^{\circ} \mathrm{C}$.

Methanol (MeOH, LC-MS grade), ACN (LC-MS grade), formic acid (Fac, HPLC grade), aqueous solution of ammonium hydroxide ( $25 \%$, HPLC grade), and ammonium formate (HPLC grade) were obtained from Fisher Scientific (Leicestershire, UK). Water (LC-MS grade) was purchased from Merck (Darmstadt, Germany). Beta-glucuronidase (Type IX from E. coli) was purchased from Sigma-Aldrich (St. Louis, MO, USA). Monopotassium phosphate and dipotassium phosphate were obtained from Acros Organics (Geel, Belgium). All other chemicals and reagents used were of analytical grade or better. An enzyme solution containing $2000 \mathrm{U} / \mathrm{mL} \beta$-glucuronidase was prepared fresh in phosphate buffer (75 mM, pH 6.8). The Oasis HLB SPE columns (6cc, $200 \mathrm{mg}$ ) were purchased from Waters (Milford, MA, USA).

\subsection{Samples}

Mid-stream urine samples were collected in the morning from 60 healthy volunteers (4-70 years, 26 males and 34 females) in Beijing province, immediately transferred to the laboratory and stored at $-80^{\circ} \mathrm{C}$. The ethics committee of China National Center for Food Safety Risk Assessment approved the study (No. 2018007, 15 Mar 2018), and the methods were performed according to the approved guidelines and regulations. All the participants were completely informed of the intent of this research. Informed written consents were obtained from the adult participants or parents on behalf of their children prior to inclusion in the study.

\subsection{Preparation of Standard Soultions and Quality Control Samples}

Serial calibration standard solutions at levels ranged from 0.001 to $500 \mathrm{ng} / \mathrm{mL}$ for different analytes (Table 3) were prepared in ACN/0.2\%FAc (50/50, v/v) by diluting the mixed standard solution in series. Each calibration standard solution contained $2 \mathrm{ng} / \mathrm{mL}{ }^{13} \mathrm{C}-\mathrm{OTA}, 2 \mathrm{ng} / \mathrm{mL}{ }^{13} \mathrm{C}-\mathrm{FB}_{1}, 1 \mathrm{ng} / \mathrm{mL}$ ${ }^{13} \mathrm{C}_{-} \mathrm{FB}_{2}, 1 \mathrm{ng} / \mathrm{mL}^{13} \mathrm{C}_{-} \mathrm{FB}_{3}, 30 \mathrm{ng} / \mathrm{mLTeA}^{-\mathrm{d}_{13}}, 10 \mathrm{ng} / \mathrm{mL} \mathrm{AOH}-\mathrm{d}_{2}, 1 \mathrm{ng} / \mathrm{mL} \mathrm{AME}-\mathrm{d}_{3}$, and $2 \mathrm{ng} / \mathrm{mL}$ TEN- $d_{3}$. They were prepared fresh as needed. Quality control (QC) samples were obtained by spiking analyte-free urine with the above standard mixtures to reach three (low, medium, and high) 
concentrations the same as in Table 4. QC samples were measured in each batch of test samples, and their measured values should be within $\pm 15 \%$ of the theoretical measurement values.

\subsection{Sample Preparation}

Prior to the extraction of mycotoxins in urine, urine samples were thawed at room temperature and centrifuged at $6000 \mathrm{rpm}$ for $15 \mathrm{~min}$ at $4{ }^{\circ} \mathrm{C}$. Fifty microliters of each mixed IS solution were added to $5 \mathrm{~mL}$ of the supernatant. The supernatant was incubated with $5 \mathrm{~mL}$ phosphate buffer $(75 \mathrm{mM}, \mathrm{pH} 6.8)$ containing $\beta$-glucuronidase $(2000 \mathrm{U} / \mathrm{mL})$ in a water bath with gentle shaking for $16 \mathrm{~h}$ at $37^{\circ} \mathrm{C}$ to allow digestion of mycotoxin conjugates. The mixture was cooled to room temperature and centrifuged again (9500 rpm, $10 \mathrm{~min}, 4^{\circ} \mathrm{C}$ ). The $\mathrm{pH}$ of the supernatant was adjusted to approximately 3 with formic acid and vortexed for $30 \mathrm{~s}$. The processed sample solutions were loaded onto Oasis HLB SPE columns for further purification. The columns were equilibrated with $5 \mathrm{~mL}$ methanol, $5 \mathrm{~mL}$ acetonitrile and $5 \mathrm{~mL}$ water in turn before sample loading. The column was washed with $5 \mathrm{~mL}$ of methanol/water (20/80, $\mathrm{v} / \mathrm{v}$ ), and analytes were eluted sequentially with $5 \mathrm{~mL}$ methanol and then $5 \mathrm{~mL}$ acetonitrile. The entire $10 \mathrm{~mL}$ eluent was dried under nitrogen at $40{ }^{\circ} \mathrm{C}$, reconstituted in $1 \mathrm{~mL}$ acetonitrile/water containing $0.2 \%$ FAc $(50 / 50, v / v)$, vortexed for $60 \mathrm{~s}$ and centrifuged $\left(4{ }^{\circ} \mathrm{C}, 20,000 \mathrm{rpm}, 30 \mathrm{~min}\right)$. Finally, $200 \mu \mathrm{L}$ of the supernatant was transferred to vials for LC-MS/MS analysis. The sample preparation procedure resulted in a 5-fold enrichment of the analytes. To determine free forms of mycotoxins, $5 \mathrm{~mL}$ of the supernatant from urine sample was diluted with phosphate buffer $(75 \mathrm{mM}, \mathrm{pH}$ 6.8) of equal volume. This mixture was not combined with $\beta$-glucuronidase. The remaining pre-treatment steps are the same as above for total mycotoxins.

\subsection{LC-MS/MS Conditions}

Instrumental method development and practical application for sample analysis were carried out using an Exion LC AD ${ }^{\mathrm{TM}}$ System (AB SCIEX, Concord, ON, Canada), coupled with a Sciex Triple Quad ${ }^{\circledR} 6500+$ LC-MS/MS system equipped with a Turbo V electrospray ionization (ESI) source. The Analyst (version 1.6.3) and MultiQuant ${ }^{\circledR}$ 3.0.2 software programs (AB SCIEX, Concord, ON, Canada) were used for instrument control and data evaluation, respectively.

\subsubsection{Chromatographic Conditions}

Using a gradient elution, analytes of interest were separated on a UHPLC column (CORTECS ${ }^{\mathrm{TM}}$ C18, $2.1 \times 100 \mathrm{~mm}, 1.6 \mu \mathrm{m}$, Waters, Milford, MA, USA). In order to obtain higher sensitivity for each compound, all the compounds analyzed were divided into two groups with different $\mathrm{pH}$ values. The first group consisted of $\mathrm{FB}_{1}, \mathrm{FB}_{2}, \mathrm{FB}_{3}, \mathrm{HFB}_{1}, \mathrm{HFB}_{2}, \mathrm{OTA}$, and $\mathrm{OTB}$, and the mobile phase consisted of solvent a (water acidified with $0.2 \% \mathrm{FAc}$ ) and solvent $\mathrm{B}(\mathrm{MeOH} / \mathrm{ACN}, 50 / 50, \mathrm{v} / \mathrm{v})$. After an incipient period of $1.0 \mathrm{~min}$ at $10 \% \mathrm{~B}, \mathrm{~B}$ increased to $90 \%$ within $1-4 \mathrm{~min}$, followed by a hold time of $1.9 \mathrm{~min}$. Finally, eluent B reduced to $10 \%$ within $0.1 \mathrm{~min}$, and the column was re-equilibrated at $90 \%$ a for $2 \mathrm{~min}$. The total run time was $9 \mathrm{~min}$. For the second group of compounds, acetonitrile and aqueous solution containing $5 \mathrm{mmol} / \mathrm{L}$ ammonia acetate and $0.01 \%$ ammonium hydroxide were used as mobile phases a and B, respectively. The gradient program was as follows: $10 \% \mathrm{~B}$ at $0-1.0 \mathrm{~min}, 10 \%-100 \% \mathrm{~B}$ at $1.0-5.5 \mathrm{~min}, 100 \% \mathrm{~B}$ at $5.5-7.5 \mathrm{~min}$, and $10 \% \mathrm{~B}$ at $7.6-10 \mathrm{~min}$. The column temperature was $40{ }^{\circ} \mathrm{C}$, and the flow rate was $0.4 \mathrm{~mL} / \mathrm{min}$. The injection volume was $5 \mu \mathrm{L}$.

\subsubsection{Mass Spectrometry Condition}

Both positive and negative ionization modes were run simultaneously by quickly switching polarity during the analysis. ESI-MS/MS analysis in scheduled multiple reaction-monitoring (sMRM) mode allowed for quantification of these compounds, using internal standards. Analyte-related MS/MS parameters were optimized via directly injection of individual standard via a peristaltic pump. Parameters of all conducted measurements are displayed in Tables 1 and 2. 


\subsection{Method Validation}

The established method (involving enzyme hydrolysis, SPE purification, and UHPLC-MS/MS analysis) was validated to assess its performance according to Commission Decision 2002/657/EC [46] and the guidelines of European Medicines Agency (EMEA) [47] and Food and Drug Administration (FDA) [48]. The validation was conducted for each of the 18 target mycotoxins, and the main parameters evaluated included linearity, method recovery, precision (intra and inter-day variability), LOD and LOQ.

The linearity was assessed in the range from the LOQ up to $25-500 \mathrm{ng} / \mathrm{mL}$ for different analytes by analyzing calibration standards at ten concentrations on three consecutive days, using linear regression with $1 / x$ weighting. LOD and LOQ of each analyte in urine was evaluated by spiking blank urine matrix at low concentration levels that generated signal-to-noise ratios $(\mathrm{S} / \mathrm{N})$ of 3 and 10, respectively. Method recovery $\left(R_{M}\right)$ and precision were investigated at low (0.05-1 ng/mL for different analytes), medium (0.5-10 ng/mL), and high (2-40 ng/mL) spiking levels in blank urine in six replicates with internal standards correction. Evaluation of inter-day precision was performed on three different days. Relative standard deviation (RSD) was calculated to represent precision.

Matrix effects (SSE), extraction recovery $\left(R_{E}\right)$, and apparent recovery $\left(R_{A}\right)$ were evaluated by three sets of calibration curves without internal standard correction [49]: Matrix-matched calibration curves prepared by spiking mycotoxin standard solutions into blank urine before (A) and after (B) sample preparation and a calibration curve prepared in ACN/0.2\%FAc (50/50) (C). Each calibration curve consisted of five concentration levels in three replicates each. Curve B and C were directly injected for UHPLC-MS/MS analysis. Curve a was subjected to the whole sample preparation procedure prior to instrument analysis, which lead to a 5-fold enrichment of analytes. Therefore, the spiking levels of curve B and C (ranged 0.05-200 ng/mL) were 5 times higher than that of curve a (ranged 0.01-40 ng/mL) to achieve the same theoretical concentration in measurement solutions. The values of SSE, $R_{A}$, and $R_{E}$ for the analytical methods were calculated using the following formulas:

$$
\begin{aligned}
& \text { SSE }(\%)=(\text { slope of calibration curve B)/(slope of calibration curve } C), \\
& R_{E}(\%)=(\text { slope of calibration curve } A) /(\text { slope of calibration curve } B), \\
& R_{A}(\%)=(\text { slope of calibration curve } A) /(\text { slope of standard curve } C)
\end{aligned}
$$

Author Contributions: Methodology, Z.L. and S.Z.; validation, Z.L. and S.Z.; formal analysis, Z.L., L.W. and X.Z.; investigation, S.Z.; writing-original draft preparation, Z.L. and X.Z.; writing-review and editing, S.Z. and L.W.; supervision, Z.G. and Y.Z.; project administration, S.Z and Y.Z.; funding acquisition, S.Z. and Y.W. All authors have read and agreed to the published version of the manuscript.

Funding: This research was funded by the National Natural Science Foundation of China (31871723), National Key Research and Development Program of China (2018YFC1602600), CFSA “523” High Level Talents Development Project, and Food Safety Research Unit (2019RU014) of Chinese Academy of Medical Science.

Conflicts of Interest: The authors declare no conflict of interest.

\section{References}

1. Freire, L.; Sant'Ana, A.S. Modified mycotoxins: An updated review on their formation, detection, occurrence, and toxic effects. Food Chem. Toxicol. 2018, 111, 189-205. [PubMed]

2. Da Rocha, M.E.B.; Freire, F.D.C.O.; Maia, F.E.F.; Guedes, M.I.F.; Rondina, D. Mycotoxins and their effects on human and animal health. Food Control. 2014, 36, 159-165. [CrossRef]

3. Sarkanj, B.; Ezekiel, C.N.; Turner, P.C.; Abia, W.A.; Rychlik, M.; Krska, R.; Sulyok, M.; Warth, B. Ultra- sensitive, stable isotope assisted quantification of multiple urinary mycotoxin exposure biomarkers. Anal. Chim. Acta 2018, 1019, 84-92. 
4. Warth, B.; Sulyok, M.; Fruhmann, P.; Mikula, H.; Berthiller, F.; Schuhmacher, R.; Hametner, C.; Abia, W.A.; Adam, G.; Frohlich, J.; et al. Development and validation of a rapid multi-biomarker liquid chromatography/tandem mass spectrometry method to assess human exposure to mycotoxins. Rapid Commun. Mass. Spectrom. 2012, 26, 1533-1540.

5. Cao, X.; Li, X.; Li, J.; Niu, Y.; Shi, L.; Fang, Z.; Zhang, T.; Ding, H. Quantitative determination of carcinogenic mycotoxins in human and animal biological matrices and animal-derived foods using multi-mycotoxin and analyte-specific high performance liquid chromatography-tandem mass spectrometric methods. J. Chromatogr. B 2018, 1073, 191-200.

6. Marín, S.; Cano-Sancho, G.; Sanchis, V.; Ramos, A.J. The role of mycotoxins in the human exposome: Application of mycotoxin biomarkers in exposome-health studies. Food Chem. Toxicol. 2018, 121, 504-518. [CrossRef]

7. Serrano, A.; Capriotti, A.; Cavaliere, C.; Piovesana, S.; Samperi, R.; Ventura, S.; Laganà, A. Development of a Rapid LC-MS/MS Method for the Determination of Emerging Fusarium mycotoxins Enniatins and Beauvericin in Human Biological Fluids. Toxins 2015, 7, 3554-3571. [CrossRef]

8. Fan, K.; Xu, J.; Jiang, K.; Liu, X.; Meng, J.; Di Mavungu, J.D.; Guo, W.; Zhang, Z.; Jing, J.; Li, H.; et al. Determination of multiple mycotoxins in paired plasma and urine samples to assess human exposure in Nanjing, China. Environ. Pollut. 2019, 248, 865-873. [CrossRef]

9. FAO. Worldwide Regulations for Mycotoxins in Food and Feed in 2003; FAO Food and Nutrition Paper No. 81; Food and Agriculture Organization of the United Nations: Rome, Italy, 2004.

10. Commission of the European Communities. Commission Regulation (EC) No 1881/2006 of 19 December 2006 Setting Maximum Levels for Certain Contaminants in Foodstuffs; Commission of the European Communities: Brussel, Belgium, 2006; Available online: https://eur-lex.europa.eu/LexUriServ/LexUriServ.do?uri=OJ:L:2006: 364:0005:0024:EN:PDF (accessed on 16 March 2020).

11. EFSA. Dietary exposure assessment to Alternaria toxins in the European population. EFSA J. 2016, 14, 04654.

12. EFSA Panel on Contaminants in the Food Chain (CONTAM). Scientific opinion on the risks to human and animal health related to the presence of beauvericin and enniatins in food and feed. EFSA J. 2014, 12, 3802. [CrossRef]

13. Warth, B.; Sulyok, M.; Krska, R. LC-MS/MS-based multibiomarker approaches for the assessment of human exposure to mycotoxins. Anal. Bioanal. Chem. 2013, 405, 5687-5695. [CrossRef]

14. Ediage, E.N.; Di Mavungu, J.D.; Song, S.; Wu, A.; Van Peteghem, C.; De Saeger, S. a direct assessment of mycotoxin biomarkers in human urine samples by liquid chromatography tandem mass spectrometry. Anal. Chim. Acta 2012, 741, 58-69. [CrossRef]

15. Martins, C.; Vidal, A.; De Boevre, M.; De Saeger, S.; Nunes, C.; Torres, D.; Goios, A.; Lopes, C.; Assuncao, R.; Alvito, P. Exposure assessment of Portuguese population to multiple mycotoxins: The human biomonitoring approach. Int. J. Hyg. Environ. Health 2019, 222, 913-925. [CrossRef]

16. Vidal, A.; Cano-Sancho, G.; Marín, S.; Ramos, A.J.; Sanchis, V. Multidetection of urinary ochratoxin A, deoxynivalenol and its metabolites: Pilot time-course study and risk assessment in Catalonia, Spain. World Mycotoxin J. 2016, 9, 597-612. [CrossRef]

17. Lauwers, M.; De Baere, S.; Letor, B.; Rychlik, M.; Croubels, S.; Devreese, M. Multi LC-MS/MS and LC-HRMS Methods for Determination of 24 Mycotoxins including Major Phase I and II Biomarker Metabolites in Biological Matrices from Pigs and Broiler Chickens. Toxins 2019, 11, 171. [CrossRef]

18. Heyndrickx, E.; Sioen, I.; Huybrechts, B.; Callebaut, A.; De Henauw, S.; De Saeger, S. Human biomonitoring of multiple mycotoxins in the Belgian population: Results of the BIOMYCO study. Environ. Int. 2015, 84, 82-89. [CrossRef]

19. Franco, L.T.; Petta, T.; Rottinghaus, G.E.; Bordin, K.; Gomes, G.A.; Alvito, P.; Assuncao, R.; Oliveira, C.A.F. Assessment of mycotoxin exposure and risk characterization using occurrence data in foods and urinary biomarkers in Brazil. Food Chem. Toxicol. 2019, 128, 21-34. [CrossRef]

20. Klapec, T.; Sarkanj, B.; Banjari, I.; Strelec, I. Urinary ochratoxin a and ochratoxin alpha in pregnant women. Food Chem. Toxicol. 2012, 50, 4487-4492. [CrossRef]

21. Munoz, K.; Blaszkewicz, M.; Degen, G.H. Simultaneous analysis of ochratoxin a and its major metabolite ochratoxin alpha in plasma and urine for an advanced biomonitoring of the mycotoxin. J. Chromatogr. B 2010, 878, 2623-2629. [CrossRef] 
22. De Girolamo, A.; Lattanzio, V.M.; Schena, R.; Visconti, A.; Pascale, M. Use of liquid chromatography -high-resolution mass spectrometry for isolation and characterization of hydrolyzed fumonisins and relevant analysis in maize-based products. J. Mass Spectrom. 2014, 49, 297-305. [CrossRef]

23. Cawood, M.; Gelderblom, W.C.; Alberts, J.F.; Snyman, S.D. Mycotoxin Biomarkers of Exposure: a Comprehensive Review. Comprehensive Reviews in Food Science and Food Safety. Food Chem. Toxicol. 1994, 32, 627-632. [CrossRef]

24. Gambacorta, S.; Solfrizzo, H.; Visconti, A.; Powers, S.; Cossalter, A.M.; Pinton, P.; Oswald, I.P. Validation study on urinary biomarkers of exposure for aflatoxin B1, ochratoxin A, fumonisin B1, deoxynivalenol and zearalenone in piglets. World Mycotoxin J. 2013, 6, 299-308. [CrossRef]

25. Souto, P.; Jager, A.V.; Tonin, F.G.; Petta, T.; Di Gregorio, M.C.; Cossalter, A.M.; Pinton, P.; Oswald, I.P.; Rottinghaus, G.E.; Oliveira, C.A.F.; et al. Determination of fumonisin B1 levels in body fluids and hair from piglets fed fumonisin B1-contaminated diets. Food Chem. Toxicol. 2017, 108, 1-9. [CrossRef]

26. Vidal, A.; Mengelers, M.; Yang, S.; De Saeger, S.; De Boevre, M. Mycotoxin Biomarkers of Exposure: a Comprehensive Review. Compr. Rev. Food Sci. Food Saf. 2018, 17, 1127-1155. [CrossRef]

27. Shephard, G.; Thiel, P.G.; Sydenham, E.W.; Vleggaar, R.; Alberts, J.F. Determination of the mycotoxin fumonisin B1 and identification of its partially hydrolysed metabolites in the faeces of non-human primates. Food Chem. Toxicol. 1994, 32, 23-29. [CrossRef]

28. Gordon, S.; Shephard, P.G.T.; Sydenham, E.W.; Savard, M.E. Fate of a single dose of [14C]-labelled fumonisin B1 in vervet monkeys. Nat. Toxins 1995, 3, 145-150.

29. Berthiller, F.; Crews, C.; Dall'Asta, C.; Saeger, S.D.; Haesaert, G.; Karlovsky, P.; Oswald, I.P.; Seefelder, W.; Speijers, G.; Stroka, J.; et al. Masked mycotoxins: a review. Mol. Nutr. Food Res. 2013, 57, 165-186. [CrossRef]

30. Righetti, L.; Fenclova, M.; Dellafiora, L.; Hajslova, J.; Stranska-Zachariasova, M.; Dall'Asta, C. High resolution-ion mobility mass spectrometry as an additional powerful tool for structural characterization of mycotoxin metabolites. Food Chem. 2018, 245, 768-774. [CrossRef]

31. Duarte, S.C.; Lino, C.M.; Pena, A. Ochratoxin a in food and urine: a nationwide Portuguese two-year study. World Mycotoxin J. 2015, 8, 121-132. [CrossRef]

32. Ali, N.; Munoz, K.; Degen, G.H. Ochratoxin a and its metabolites in urines of German adults-An assessment of variables in biomarker analysis. Toxicol. Lett. 2017, 275, 19-26. [CrossRef]

33. Rodriguez-Carrasco, Y.; Molto, J.C.; Manes, J.; Berrada, H. Development of a GC-MS/MS strategy to determine 15 mycotoxins and metabolites in human urine. Talanta 2014, 128, 125-131. [CrossRef]

34. Bordin, K.; Rottinghaus, G.E.; Landers, B.R.; Ledoux, D.R.; Kobashigawa, E.; Corassin, C.H.; Oliveira, C.A.F. Evaluation of fumonisin exposure by determination of fumonisin B1 in human hair and in Brazilian corn products. Food Control. 2015, 53, 67-71. [CrossRef]

35. Slobodchikova, I.; Vuckovic, D. Liquid chromatography-High resolution mass spectrometry method for monitoring of 17 mycotoxins in human plasma for exposure studies. J. Chromatogr. A 2018, 1548, 51-63. [CrossRef]

36. De Santis, B.; Raggi, M.E.; Moretti, G.; Facchiano, F.; Mezzelani, A.; Villa, L.; Bonfanti, A.; Campioni, A.; Rossi, S.; Camposeo, S.; et al. Study on the Association among Mycotoxins and other Variables in Children with Autism. Toxins 2017, 9, 203. [CrossRef]

37. Ezekiel, C.N.; Warth, B.; Ogara, I.M.; Abia, W.A.; Ezekiel, V.C.; Atehnkeng, J.; Sulyok, M.; Turner, P.C.; Tayo, G.O.; Krska, R.; et al. Mycotoxin exposure in rural residents in northern Nigeria: a pilot study using multi-urinary biomarkers. Environ. Int. 2014, 66, 138-145. [CrossRef]

38. Rodriguez-Carrasco, Y.; Izzo, L.; Gaspari, A.; Graziani, G.; Manes, J.; Ritieni, A. Urinary levels of enniatin B and its phase I metabolites: First human pilot biomonitoring study. Food Chem. Toxicol. 2018, 118, 454-459. [CrossRef]

39. Solfrizzo, M.; Gambacorta, L.; Lattanzio, V.M.T.; Powers, S.; Visconti, A. Simultaneous LC-MS/MS determination of aflatoxin M1, ochratoxin A, deoxynivalenol, de-epoxydeoxynivalenol, $\alpha$ and $\beta$-zearalenols and fumonisin B1 in urine as a multi-biomarker method to assess exposure to mycotoxins. Anal. Bioanal. Chem. 2011, 401, 2831-2841. [CrossRef]

40. Gazzotti, T.; Zironi, E.; Lugoboni, B.; Barbarossa, A.; Piva, A.; Pagliuca, G. Analysis of fumonisins B1, B2 and their hydrolysed metabolites in pig liver by LC-MS/MS. Food Chem. 2011, 125, 1379-1384. [CrossRef] 
41. Huybrechts, B.; Martins, J.C.; Debongnie, P.; Uhlig, S.; Callebaut, A. Fast and sensitive LC-MS/MS method measuring human mycotoxin exposure using biomarkers in urine. Arch. Toxicol. 2015, 89, 1993-2005. [CrossRef]

42. Duarte, S.; Bento, J.; Pena, A.; Lino, C.M.; Delerue-Matos, C.; Oliva-Teles, T.; Morais, S.; Correia, M.; Oliveira, M.B.; Alves, M.R.; et al. Monitoring of ochratoxin a exposure of the Portuguese population through a nationwide urine survey-Winter 2007. Sci. Total Environ. 2010, 408, 1195-1198. [CrossRef]

43. Gerding, J.; Ali, N.; Schwartzbord, J.; Cramer, B.; Brown, D.L.; Degen, G.H.; Humpf, H.U. a comparative study of the human urinary mycotoxin excretion patterns in Bangladesh, Germany, and Haiti using a rapid and sensitive LC-MS/MS approach. Mycotoxin Res. 2015, 31, 127-136. [CrossRef]

44. Asam, S.; Habler, K.; Rychlik, M. Determination of tenuazonic acid in human urine by means of a stable isotope dilution assay. Anal. Bioanal. Chem. 2013, 405, 4149-4158. [CrossRef]

45. Sun, D.; Qiu, N.; Zhou, S.; Lyu, B.; Zhang, S.; Li, J.; Zhao, Y.; Wu, Y. Development of Sensitive and Reliable UPLC-MS/MS Methods for Food Analysis of Emerging Mycotoxins in China Total Diet Study. Toxins 2019, 11, 166. [CrossRef]

46. Commission Decision 2002/657/EC Implementing Council Directive 96/23/EC Concerning the Performance of Analytical Methods and the Interpretation of Results. Available online: https://eur-lex.europa.eu/LexUriServ/ LexUriServ.do?uri=OJ:L:2002:221:0008:0036:EN:PDF (accessed on 17 March 2019).

47. European Medicines Agency. Guideline on Bioanalytical Method Validation. Available online: http://www.ema.europa.eu/docs/en_GB/document_library/Scientific_guideline/2011/08/WC500109686. pdf (accessed on 12 January 2016).

48. FDA. FDA Guidance for Industry: Bioanalytical Method Validation; Food and Drug Administration: Silver Spring, MD, USA, 2001. Available online: https:/www.fda.gov/downloads/Drugs/ GuidanceComplianceRegulatoryInformation/Guidances/UCM070107.pdf (accessed on 16 March 2019).

49. Matuszewski, B.K.; Constanzer, M.L.; Chavez-Eng, C.M. Strategies for the assessment of matrix effect in quantitative bioanalytical methods based on HPLC-MS/MS. Anal. Chem. 2003, 75, 3019-3030. [CrossRef]

(C) 2020 by the authors. Licensee MDPI, Basel, Switzerland. This article is an open access article distributed under the terms and conditions of the Creative Commons Attribution (CC BY) license (http://creativecommons.org/licenses/by/4.0/). 